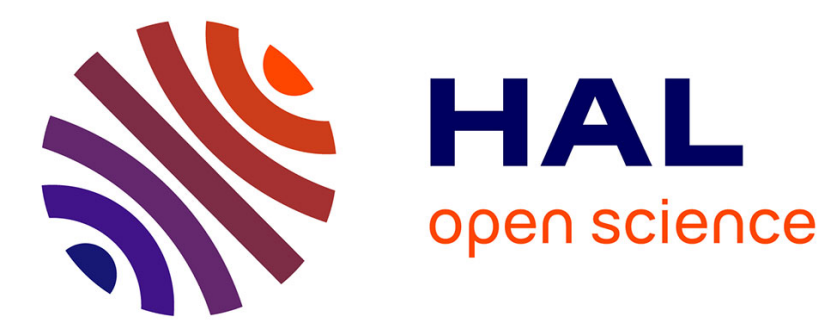

\title{
Three-dimensional instability and vorticity patterns in the wake of a flat plate
}

Stéphanie Julien, J Lasheras, Jean-Marc Chomaz

\section{To cite this version:}

Stéphanie Julien, J Lasheras, Jean-Marc Chomaz. Three-dimensional instability and vorticity patterns in the wake of a flat plate. Journal of Fluid Mechanics, 2003, 479 (march), pp.155-189. 10.1017/s0022112002003580 . hal-01024925

\section{HAL Id: hal-01024925}

https: / hal-polytechnique.archives-ouvertes.fr/hal-01024925

Submitted on 3 Sep 2014

HAL is a multi-disciplinary open access archive for the deposit and dissemination of scientific research documents, whether they are published or not. The documents may come from teaching and research institutions in France or abroad, or from public or private research centers.
L'archive ouverte pluridisciplinaire HAL, est destinée au dépôt et à la diffusion de documents scientifiques de niveau recherche, publiés ou non, émanant des établissements d'enseignement et de recherche français ou étrangers, des laboratoires publics ou privés. 


\title{
Three-dimensional instability and vorticity patterns in the wake of a flat plate
}

\author{
By STEPHANIE JULIEN ${ }^{1,2}$, JUAN LASHERAS \\ AND JEAN-MARC CHOMAZ \\ ${ }^{1}$ Department of Mechanical and Aerospace Engineering, University of California, San Diego, \\ La Jolla, CA 92093-0411, USA \\ ${ }^{2}$ Laboratoire d'Hydrodynamique, CNRS-UMR 156, École Polytechnique, \\ F-91128 Palaiseau Cedex, France
}

(Received 15 April 2002 and in revised form 1 October 2002)

We investigated experimentally the dynamics of the three-dimensional secondary instability developing in the wake of a thin flat plate at moderate Reynolds numbers. The wake is formed as the two laminar boundary layers developing on each side merge at the trailing edge of the flat plate. Both the spatial and temporal evolution of the two- and three-dimensional instabilities are analysed by means of laser-induced visualizations of the deformation of the interface separating the two streams. It was found that although the wake may exhibit two distinct three-dimensional modes with different symmetry characteristics, Modes 1 and 2 (Lasheras \& Meiburg 1990), the latter appears to be amplified first, thereafter dominating the evolution of the near wake. By varying the forcing frequency of the primary two-dimensional instability, we found that the wavelength of the three-dimensional mode is selected by the wavelength of the two-dimensional Kármán vortices, with a ratio $\left(\lambda_{3 D} / \lambda_{2 D}\right)$ of order one. In the far-wake region, both modes appear to grow and co-exist. Furthermore, by analysing the response of the wake to spanwise-periodic and impulsive perturbations applied at the trailing edge of the plate, we demonstrate that the nature of the secondary instability of the wake behind a thin flat plate is convective. In addition, both modes are shown to have comparable wavelengths and to be the result of the same instability mechanism.

\section{Introduction}

It has long been established that at moderate and large Reynolds numbers, the wake behind two-dimensional bodies exhibits coherent vortical structures composed of a staggered configuration of counter-rotating vortices, the Kármán vortex street (Roshko 1954; Taneda 1977). In addition, it has been found experimentally as well as analytically and numerically that this primary state is, itself, unstable to three-dimensional perturbations. After an initial transition period, the wake behind two-dimensional bodies has been shown to develop a three-dimensional structure. As early as 1958, Grant (1958) provided experimental evidence that the nominally two-dimensional wake behind a bluff body develops a three-dimensional vortical structure composed of counter-rotating pairs of streamwise vortices whose axes are located on planes inclined to the plane of the wake. Similar vortex pair structures were found by Payne \& Lumley (1967), Townsend (1979) and Mumford (1983) among others. Additional experimental evidence of this three-dimensional 
structure has been provided by Meiburg \& Lasheras (1987, 1988), Cimbala, Nagib \& Roshko (1988), Williamson (1988, 1989, 1996a,b), Ferre \& Girault (1989), Marasli, Champagne \& Wygnanski (1989), Eisenlohr \& Eckelmann (1989), Lasheras \& Meiburg (1990), Konig, Eisenlohr \& Eckelman (1990, 1992), Miller \& Williamson (1994), Monkewitz, Williamson \& Miller (1996), among others. A comprehensive review of the experimental evidence of the three-dimensional transition of the wake behind cylinders can be found in the recent paper by Williamson (1996b). Floquet stability analyses provided by Noack \& Eckelman (1994) and Barkley \& Henderson (1996) as well as direct numerical simulations by Braza (1994) and Persillon \& Braza (1998) have also confirmed the formation of three-dimensional vortical structures in bluff-body wakes.

The transition to three-dimensionality is thought to determine the mixing properties and transition to turbulence. Furthermore, the combined development of both twoand three-dimensional instabilities, and their receptivity to various forcing and actuation techniques are believed to play a dominant role in control strategies to minimize drag of submerged bodies or to enhance the mixing of passive scalars in the wake. Of course, the decomposition into primary and secondary instability arising sequentially is often only conceptual since in many flows both may develop simultaneously.

In order to analyse the three-dimensional transition in the wake behind a nominally two-dimensional body, and in particular to study the instability mechanisms leading to this transition, Meiburg \& Lasheras $(1987,1988)$ and Lasheras \& Meiburg $(1990)$ conducted a combined experimental and numerical investigation of the structure of the near region of the wake developing behind a flat plate at low and moderate Reynolds numbers subjected to various types of spanwise perturbations. These studies have shown that the wake behind a flat plate may develop two distinct three-dimensional vorticity modes which preserve the periodicity of the two-dimensional Kármán street and which exhibit different symmetry properties (Modes 1 and 2) $\dagger$. By forcing the wake with periodic spanwise perturbations oriented in either the cross-stream or the streamwise direction, they found that the wake develops two different threedimensional patterns. Mode 1, which was found to develop from the cross-stream perturbation, results in the formation of counter-rotating pairs of streamwise vortices that acquire a lambda-shaped structure and are located in the braids connecting consecutive Kármán vortices of opposite sign. Mode 2, which was found to evolve from the effect of periodic perturbations of the streamwise velocity in the plane of the wake, also results in the formation of counter-rotating pairs of streamwise vortices in the braids connecting consecutive Kármán vortices; however, these streamwise vortex pairs remain parallel to each other, while their separation is not uniform along the span. Meiburg \& Lasheras (1988) and Lasheras \& Meiburg (1990) confirmed that these three-dimensional patterns, which were observed in experiments, can be simulated using three-dimensional inviscid vortex dynamics methods. Based on their vortex dynamic simulations and the experimental evidence, they concluded that the mechanism responsible for the transition to three-dimensionality is common to both modes and can be described as follows: first, the primary two-dimensional instability leads to the formation of a staggered array of spanwise vortex tubes, i.e. the Kármán vortex street; second, depending on the orientation of the spanwise perturbation, counter-rotating pairs of streamwise vortex tubes are formed in the high-strain-rate

$\dagger$ Lasheras \& Meiburg also found the existence of a wide variety of additional three-dimensional modes resulting from the growth of oblique, subharmonic waves. 
braid regions; third, the Kármán vortices acquire a sinusoidal undulation resulting in either an anti-symmetric (sinuous) or symmetric (varicose) configuration. Thus, the overall mechanism common to both modes appears to be a combination of an instability due to the stretching at the stagnation point in the braid regions and an instability of the primary vortex cores. It is important to point out that the temporal secondary stability analysis of a von Kármán street resulting from the nonlinear development of a Bickley velocity profile by Sutherland \& Peltier (1994) is in very good agreement with the observations and the interpretations of Meiburg and Lasheras. In particular, their analysis shows that, in both modes, the perturbation extracts energy concomitantly from the braid and from the core regions of the flow.

Studying the wake behind cylinders, Williamson $(1988,1996 a, b)$ has also found the existence of two three-dimensional vorticity modes (referred to as Modes A and B) that resemble those found for the flat plate. Williamson also found that both modes involve the generation of streamwise vortex pairs located in the braid connecting the primary Kármán vortices. However, Williamson (1996a) and Leweke \& Williamson (1998) attributed each mode to a different type of instability mechanism. Since the spanwise wavelength of Mode A scales on the large physical features of the wake, namely the primary vortex cores, they attributed its formation to an elliptic instability of these vortices. On the other hand, they found that Mode B has a much shorter wavelength, scaling with the smaller physical length scale, namely the thickness of the braid shear layer. Leweke \& Williamson (1998) argued that this second mode, which develops at a higher Reynolds number, could be the result of an instability of the hyperbolic regions (braid regions) of the flow. These observations motivated a return to the flat-plate wake. Indeed, the early experiments of Meiburg \& Lasheras did not try to identify if the three-dimensional Modes 1 and 2, forming in the wake of the flat plate, have different preferred wavelengths nor if they appear predominantly at different Reynolds numbers. The present work extends the early experiments of Lasheras \& Meiburg and is an extensive study of the evolution of the wake behind a flat plate at Reynolds numbers of about 200 in order to answer several open questions, namely:

1. What is the preferred wavelength of each of these modes?

2. Can both three-dimensional modes coexist in the transitional regime?

3. Is the origin of the two three-dimensional patterns due to two different types of instabilities, namely elliptic or hyperbolic as argued by Leweke \& Williamson?

In particular, we have used the symmetry properties which each of these two modes possesses to identify their appearance under both unforced and forced conditions. To aid the reader in the interpretation of our results, in $\S 2$ we will first present a comprehensive summary of the topology of the three-dimensional vorticity field corresponding to each of the two modes. The experimental set-up and techniques, presented in $\S 3$, are a refinement of those used in previous experiments reported in Meiburg \& Lasheras (1988) and Lasheras \& Meiburg (1990). In particular, we introduce various image processing techniques that allow us to identify the threedimensional patterns and to determine the preferred wavelength and the growth rates of these modes. In $\S 4$, the primary two-dimensional instability is characterized. In $\S 5$, we present experiments corresponding to the near and the far region of the wake developing under unforced conditions as well as under a single wave, two-dimensional forcing. We will also present the effects of the combined two- and three-dimensional forcing and measurements of the preferred wavelength of the three-dimensional instability in this section. Finally, in $\S 6$, we present a discussion and speculatate on the three-dimensional wavelength and mode selection in plane wakes. 
(a)

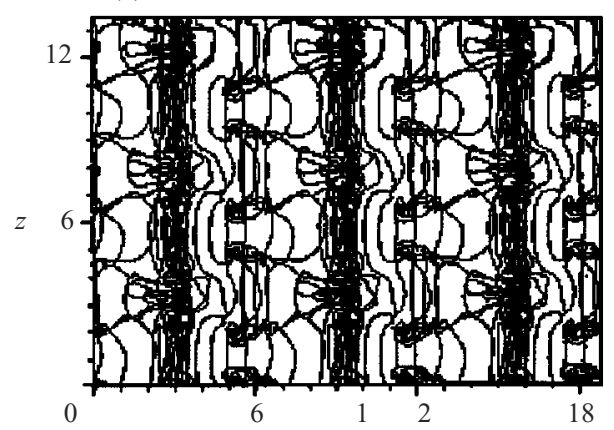

(b)

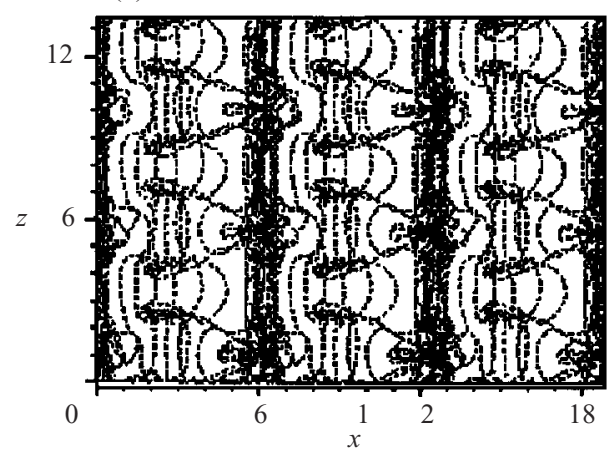

(c)

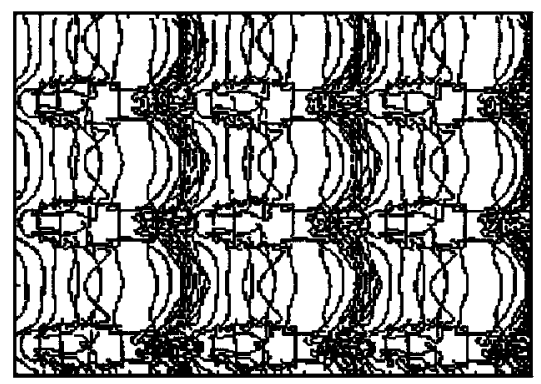

(d)



FIGURE 1. Plan view of the filaments of the upper $(a, c)$ and lower $(b, d)$ layers at a time $t=169$. $(a, b)$ Mode $1 ;(c, d)$ Mode 2 (from Lasheras \& Meiburg 1990).

\section{Symmetries and dynamics of Modes 1 and 2}

In the previous investigation (Meiburg \& Lasheras 1988; Lasheras \& Meiburg 1990), we showed that under the combined effect of both two- and three-dimensional perturbations, the overall induction of the vorticity (concentration, reorientation and stretching) results in the formation of counter-rotating pairs of streamwise vortices that are located in the braids connecting consecutive Kármán vortices of opposite sign (planes inclined to the plane of the wake). Depending on the orientation of the initial spanwise perturbation, the wake behind a flat plate was observed to develop two distinct three-dimensional vorticity modes with different symmetry properties, Meiburg \& Lasheras (1987, 1988) and Lasheras \& Meiburg (1990). The reader is referred to these early papers for a detailed discussion on the mechanism of the three-dimensional instability. In this section we will simply review the most salient features of these modes which have been used throughout this study. Figure 1 shows the results of inviscid vortex dynamics simulations taken from Lasheras \& Meiburg (1990). In this study, the wake is modelled by spanwise vortex filaments of identical circulation gathering in planes depending on the sign of their vorticity. This geometry is a discretized version of two parallel sheets with opposite vorticity and appropriate perturbations will favour one mode or the other. One can observe that under the global induction of the vorticity, the perturbation vorticity in both layers is reoriented into the direction of positive strain in the evolving strain fields created by the twodimensional spanwise vortices. When subjected to a vertical (cross-stream $y, z$-plane) initial spanwise perturbation (Mode 1), this induction results in a $180^{\circ}$ phase shift between the perturbation vorticity of the two layers (figure $1 a, b$ ), resulting in a loss 
(a)

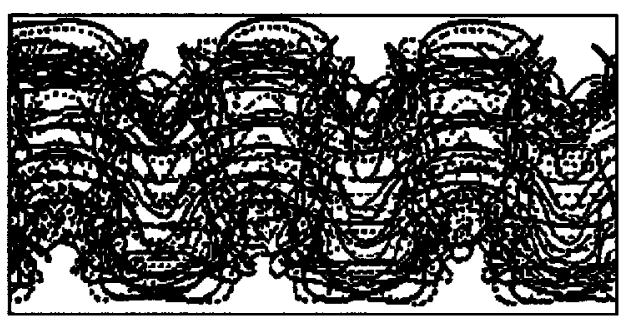

(b)

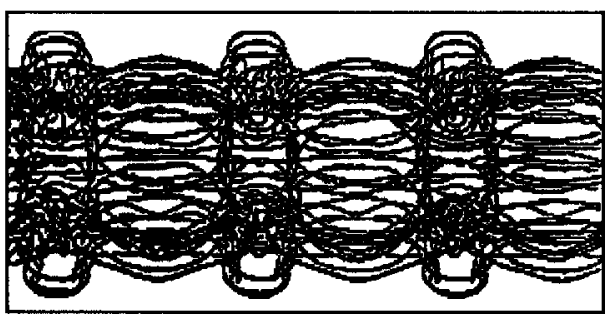

FIGURE 2. End-view ( $x, z$-plane) of the filaments of the upper and lower layers composing the wake. (a) Mode 1; (b) Mode 2 (from Lasheras \& Meiburg 1990).

of the vertical ( $x, y$-plane) symmetry of the wake. On the other hand, under the effect of a horizontal ( $x, z$-plane of the wake) initial spanwise perturbation (Mode 2), the stretched perturbation vorticity reoriented into the directions of positive strain in both layers (figure $1 c, d)$ is found to remain in phase, preserving the vertical $(x, y$-plane) symmetry of the wake.

In both cases, the formation of the Kármán vortex street has been completed and the appearance of the streamwise vorticity concentration is clearly seen in the braids. In Mode 1 (figure $1 a, b$ ), the counter-rotating pair of streamwise vortices formed in each layer acquires a characteristic lambda-shaped form, while in Mode 2 (figure $1 c, d$ ) the counter-rotating pairs of streamwise vortices of each layer always remain parallel to the $x$-direction, while their separation is not constant across the span.

In addition, the interaction of the evolving streamwise vortices with the spanwise ones leads to a vertical ( $x, y$-plane) undulation in the axis of the spanwise vortices. Figure 2 shows the end-view of all the vortex filaments comprising the upper and lower layers. Observe that the spanwise Kármán vortices are undulated in the vertical plane. In Mode 1, this undulation is in-phase between the Kármán vortices of opposite sign resulting in a sinuous shape, while in Mode 2 it is $180^{\circ}$ out-of-phase, resulting in a varicose shape. For Mode 2, it is quite remarkable that the undulation is not only vertical ( $x, y$-plane) but also in the plan view (figure $1 c, d$ ) where the symmetry is opposite, showing an in-phase undulation of the top and bottom vortices. These symmetry characteristics led us to refer interchangeably to Mode 1 as the in-phase mode or the sinuous Mode, and to Mode 2 as the out-of-phase or the varicose Mode. Due to their symmetry characteristics in the $(x, z)$-plane, we have also referred to these modes as anti-symmetric (Mode 1) and symmetric (Mode 2), following Robinson \& Saffman (1982). These are precisely the features that we have used in the present study to identify the appearance of each mode.

As an example, the perspective views of the experimentally visualized positions of the interface, corresponding to Modes 1 and 2, are presented in figure 3. The counterrotating pairs of streamwise vortices are visible, forming the lambda shape for Mode 1 and remaining parallel for Mode 2. The in-plane undulation in the spanwise vortices can also be observed for Mode 2.

\section{Experimental set-up}

The flow facility used in all the experiments described herein is a blow-down, two-stream, free surface water channel shown in figure 4. A separate supply of water for each layer is pumped into overhead tanks in which an overflow system of pipes maintains a constant head. Flow meters and valves control the gravity feed 
(a)

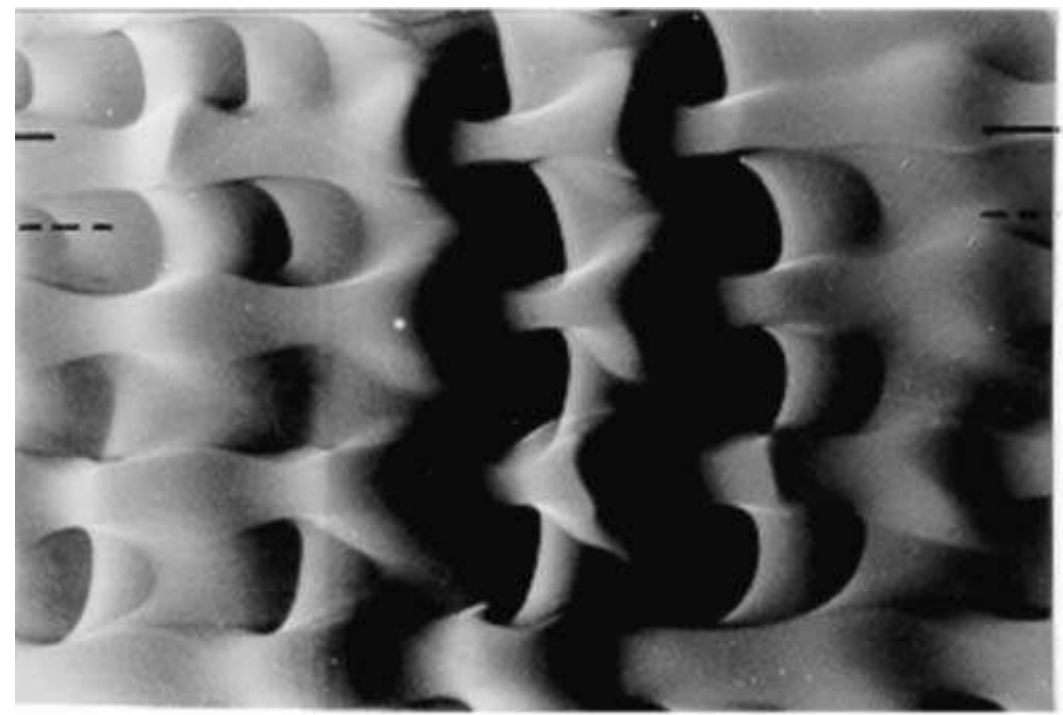

(b)

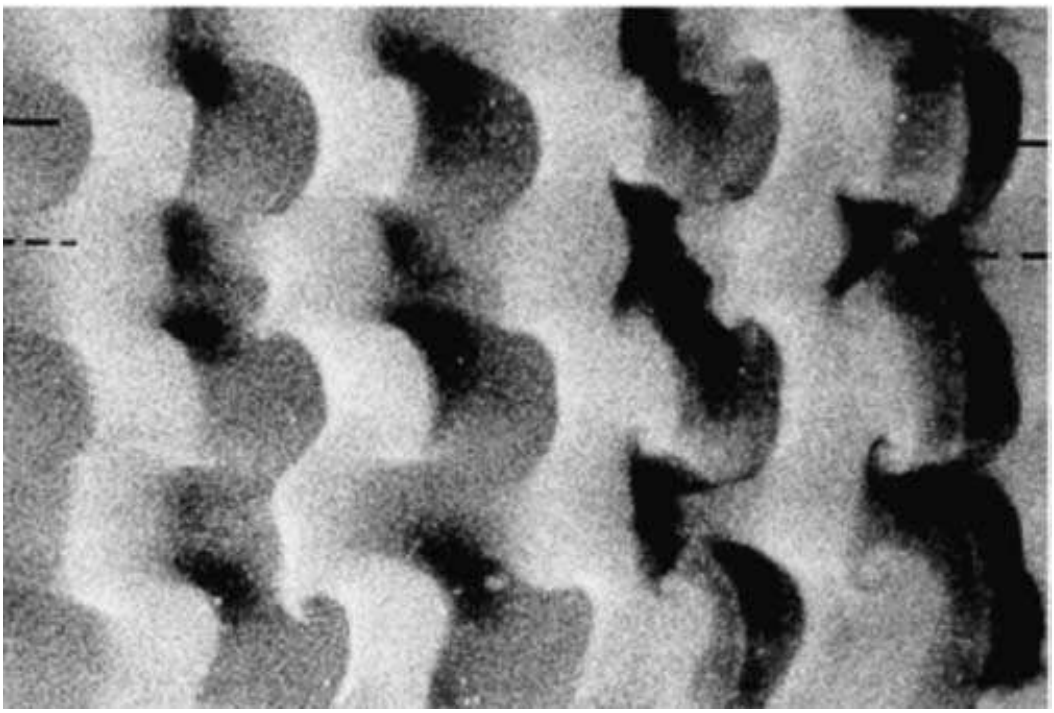

FIGURE 3. Plan views of the interface visualizations corresponding to (a) Mode 1 and (b) Mode 2. Two vertical ( $x, y$-plane) cross-cuts of the interface are also shown in each figure revealing the anti-symmetric shape in Mode 1 and the symmetric configuration of Mode 2 (from Meiburg \& Lasheras 1988).

from the overhead tanks into each of the layers. The supply to each layer passes through a settling chamber located upstream of the test section where porous foam and rubberised hair prevents surface waves from forming, decoupling the channel from any vibrations from the supply pumps. The settling chamber is followed by honeycombs which straighten the flow and by two sets of fine-mesh screens used to break down any large-scale spanwise non-uniformities in the velocity. A three-to-one 


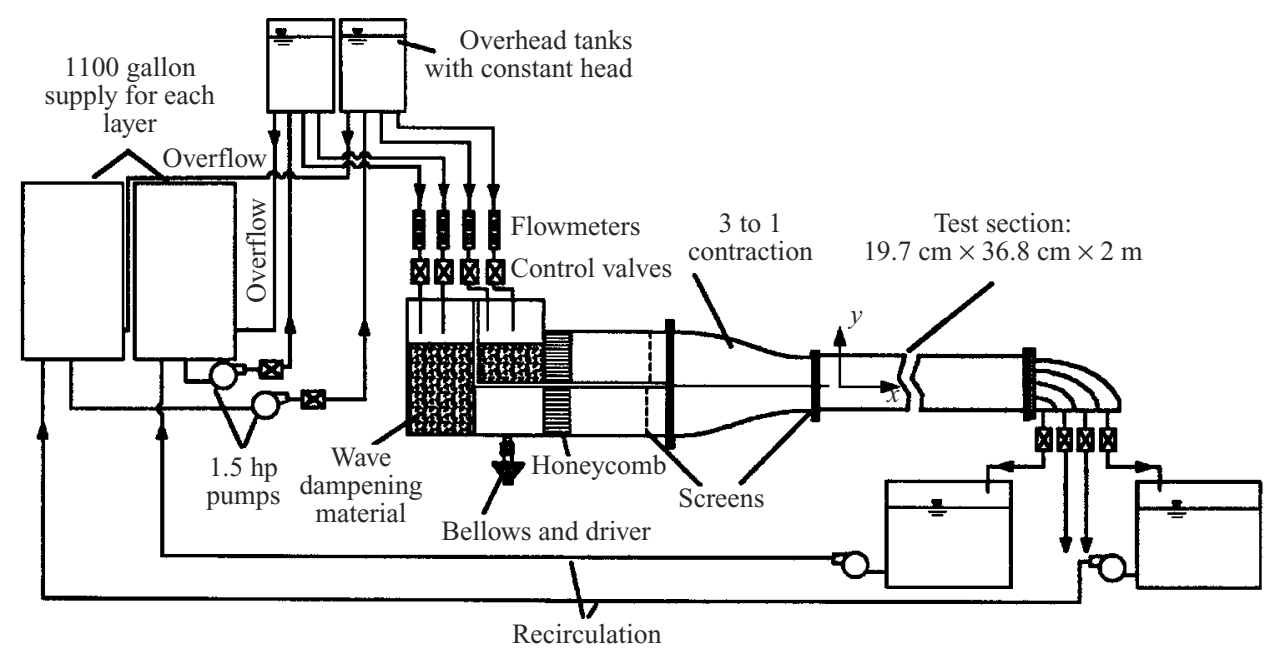

FIGURE 4. Experimental set-up.

contraction nozzle connects the settling chamber with the test section where an additional set of fine-mesh screens is used to reduce the boundary layer thickness at the entrance of the open channel test section. Effects of the wall are minimized by selecting an aspect ratio in each layer of 3.7 to 1 (horizontal to vertical). The test section has a rectangular cross-section $36.8 \mathrm{~cm}$ wide by $19.7 \mathrm{~cm}$ deep and a length of $200 \mathrm{~cm}$. This facility is a modified version of two earlier shear layer channels designed by Winant \& Browand (1974) and Lasheras, Cho \& Maxworthy (1986). A detailed description of the facility can be found in Schowalter, Van Atta \& Lasheras (1994).

The frequency of the primary spanwise Kármán vortices can be forced with a bellows and driver system as shown in figure 4. This forcing system is located in the lower layer inlet section. The driver consists of a speaker whose cone is connected to bellows. The speaker is driven by a signal generator and an amplifier with a DC offset. The speaker is fed with a sinusoidal wave of a given frequency whereby a streamwise perturbation of the axial velocity profile in the lower stream at the end of the splitter plate is generated. In all the cases reported here, the amplitude of the sinusoidal wave was such that it resulted in less than $0.5 \%$ perturbation over the mean velocity of the stream. Detailed hot-film measurements as well as flow visualizations confirmed that the forcing wave was planar at the origin of the wake (trailing edge of the splitter plate).

In the following, we will adopt a coordinate system (the same as for the figures shown in $\S 2$ ) with the origin at the edge of the splitter plate (figure 4). The $x$-axis is oriented along the streamwise direction, the $y$-axis is along the cross-stream, and the $z$-axis is in the spanwise direction. The exit section of the open channel contains four subdivisions, the flow rates in which are controlled by the globe valves shown in figure 4. Care was taken to avoid the formation of standing waves. Tests were performed to determine the sensitivity of the free streams to the setting of the exit valves. It was found that this sensitivity was very small. Once the valve settings for a certain experiment were determined, only very slight adjustments were needed to keep the level in the test section constant throughout the whole study.

The evolution of the wake was then analysed using various flow visualization techniques. Two series of experiments are reported here, corresponding to two different 


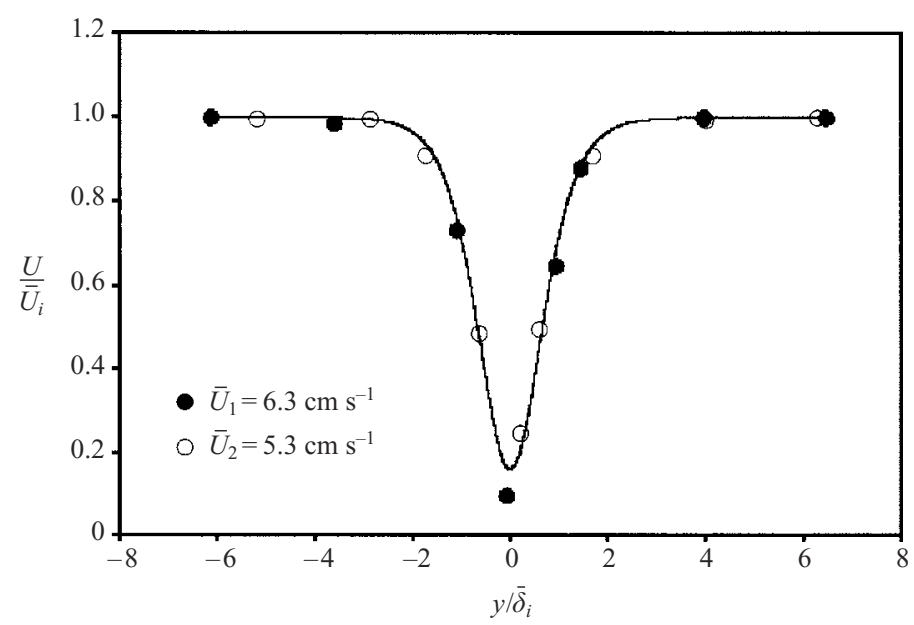

FIGURE 5. Rescaled velocity profiles measured at the trailing edge of the splitter plate, $x=2 \mathrm{~mm}$, fitted by $U / \bar{U}_{i}=1-\Theta \times \operatorname{sech}^{2}\left(y / \bar{\delta}_{i}\right)$ with $\Theta=0.8$ and $\bar{\delta}_{i}=0.88 / \sqrt{\bar{U}_{i}}$ evaluated with $10^{-2}$ accuracy.

laminar free-stream velocities, $\bar{U}_{1}=6.3 \mathrm{~cm} \mathrm{~s}^{-1}$ and $\bar{U}_{2}=5.3 \mathrm{~cm} \mathrm{~s}^{-1}$. Figure 5 shows both sets of velocities obtained using hot-film anemometry and measured at the trailing edge of the splitter plate, $x=2 \mathrm{~mm}$, with $U$ non-dimensionalized by the velocity of the free stream $\bar{U}_{i}(i \in[1 ; 2])$ and $y$ rescaled by $\bar{\delta}_{i} \propto \bar{U}_{i}^{-1 / 2}$. When scaled in this fashion, the data describe a unique curve, independent of the velocity and consistent with laminarity of the wake. The Bickley wake thicknesses are $\bar{\delta}_{1}=0.35 \mathrm{~cm}$ and $\bar{\delta}_{2}=0.38 \mathrm{~cm}$ for $\bar{U}_{1}=6.3 \mathrm{~cm} \mathrm{~s}^{-1}$ and $\bar{U}_{2}=5.3 \mathrm{~cm} \mathrm{~s}^{-1}$, respectively. The experimental conditions correspond to $R e_{1}=220$ and $R e_{2}=200\left(R e_{i}=\bar{U}_{i} \bar{\delta}_{i} / \nu\right.$, where $v$ is the kinetic viscosity of the water). They were free from the effects of the free surface and confinement. A third velocity $\bar{U}_{3}=4.8 \mathrm{~cm} \mathrm{~s}^{-1}$, was also tested less exhaustively; it confirms all the observations presented above. The downstream evolution of the velocity profiles was found to be consistent with the well-known classical result of Schlichting (1930) and Roshko (1954).

In both cases reported here, both streams were laminar with non-detectable r.m.s. velocity fluctuations. Our hot-film velocity measurements revealed not only a temporal uniformity of the flow in both streams but also a spanwise homogeneity of the velocity profile, with variations less than $0.5 \%$. Although we used de-ionized water, the flow still contained small air bubbles that uncontrollably attached to locations randomly distributed under the splitter plate. These bubbles $(<1 \mathrm{~mm}$ in diameter) resulted in uncontrollable, small-amplitude perturbations randomly distributed along the span of the flow, whose signature was undetected by the hot-wire measurements.

For each Reynolds number case, we carried out three sets of experiments: unforced or natural flow, forcing the two-dimensional primary instability only, and simultaneously forcing the two-dimensional and the three-dimensional instabilities. In each case, we used various flow visualization and digital image processing techniques to track the evolution of the wake from the trailing edge of the splitter plate to downstream distances of about $24 \mathrm{~cm}$ (always larger than 5 times the wavelength of the two-dimensional instability).

To analyse the three-dimensional evolution of the wake, we employed a technique used in the previous studies of Lasheras \& Choi (1988) and Meiburg \& Lasheras 
(1988) which consisted of visualizing the spatial and temporal deformations of the interface separating the streams. This technique allowed us to determine indirectly the growth rates of the two- and three-dimensional instabilities developing in the wake. Since this is an important element of our measurement system, we will specifically address the validity of this method later on in this section.

The evolution of the interface was tracked using planar laser-induced fluorescence (PLIF). Fluorescent dye was added to the lower layer and the position of the interface separating the two streams was recorded through the use of simultaneous longitudinal and cross-stream cuts produced by the intersection of thin laser planes. The longitudinal cut of the flow along the $(x, y)$-plane will be referred to as the 'side view', while the cross-stream cuts along planes parallel to $(y, z)$-plane performed at various downstream locations, $x$, will be referred to as 'end views'. As was shown by Schowalter et al. (1994), the use of a prism slightly submerged in the free surface of the channel allowed us to avoid any problems resulting from free surface distortion due to capillary waves and changes in the index of refraction. The camera and laser plane assemblage were systematically traversed downstream to provide the necessary information on the spatial evolution of the three-dimensional instabilities. To complement these visualizations, a spotlight arrangement, identical to the one used in Meiburg \& Lasheras (1988) to obtain the visualizations shown in figure 3, was also used to observe the evolution of the complete three-dimensional topology of the interface separating both streams.

The recorded PLIF frames were processed on a Macintosh computer using the public domain NIH Image program (developed at the US National Institutes of Health and available on the Internet at http://rsb.info.nih.gov/nih-image/). Since the characteristic time scale of diffusion of the fluorescein dye is an order of magnitude larger than the convective time scale of the flow of interest (the Schmidt number, $S c>10^{3}$ ), the interface always appears as a sharp line in our flow visualization. However, as an interface moves downstream, it becomes more diffused due to both three-dimensional foldings and the effects of light attenuation. Throughout this study, the criterion used to detect the interface was to fix its location at the position where the pixel intensity was $50 \%$ of the average value of the intensity of the dye layer. Using this method, we measured the location of the interface along the span in each digitized image (see figure 6).

The interface location was determined in a sequence of images, allowing us to obtain the complete spatial-temporal evolution of the deformed interface. This procedure was carried out for both the longitudinal laser cuts (figure $6 a$ ) and for the transverse laser cut (figure $6 b, c$ ). It is important to emphasize that due to the low convective velocity of the mean flow (5.3 to $6.3 \mathrm{~cm} \mathrm{~s}^{-1}$ ), a 30 frames per second sequence obtained with shutter speeds of the camera of $1 / 4000 \mathrm{~s}$ provided us with a very accurate description of both the spatial and temporal evolution of the interface.

Close to the splitter plate, as the two-dimensional Kármán vortex street starts to grow, the interface is always characterized on the side views by a slightly wavy line. The end-view visualization (figure $6 b$ ) reveals a very small three-dimensional deformation. Therefore, in this initial region where there is no overturning, the position of the interface, given by its elevation $h(x, z, t)((x, z)$ refers to the streamwise and the horizontal spanwise locations respectively, and $t$ to time), is a uniquely defined quantity (a single-valued function), which can be measured on either the side view (figure $6 a$ ) or the end-view cross-cut (figure $6 b$ ). Further downstream, the flow is overturned and the interface wraps around the spanwise Kármán vortices (see the downstream part of the side view shown in figure $6 a$ ). Thus, at a given location 
(a)

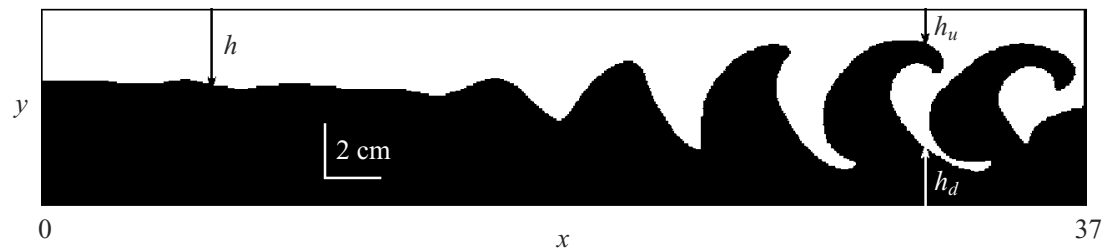

(b)

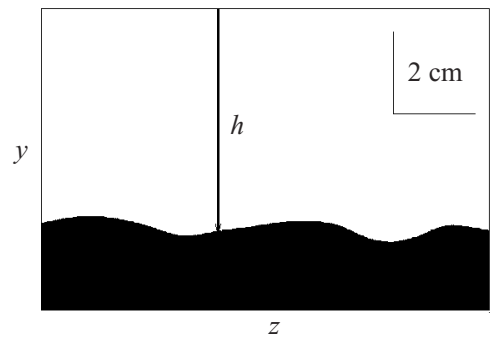

(c)

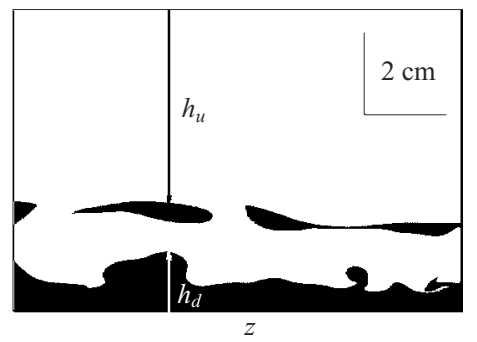

Figure 6. Interface elevation. Wake of a flat plate at $R e_{1}=220$ forced at the frequency $f=1.2 \mathrm{~Hz}(S t=\bar{\delta} f / \bar{U}=0.07):(a)$ longitudinal section $\left(z=z_{c}\right.$, centre of the channel $)$, (b) interface defined by a single line $(x=6 \mathrm{~cm}),(c)$ folded interface $(x=20 \mathrm{~cm})$.

downstream $(x, z)$, the interface is no longer defined by a single elevation, and the function $h(x, z, t)$ becomes multi-valued. The interface is then defined by several lines and closed loops may form as shown in the end-view cuts, figure $6(c)$. To analyse the stage where the interface has become highly corrugated, we introduced two symmetrically defined parameters $h_{u}(x, z, t)$ and $h_{d}(x, z, t)$ : they are respectively the uppermost and the lowermost positions of the interface at the location $(x, z)$ and at time $t$. Note that in the near wake, when there is no flow overturning, $h_{d}$ and $h_{u}$ are the same and equal to $h$ as defined above.

Since the deformation of the interface separating the two streams is a consequence of the growth of the two-dimensional and three-dimensional instabilities, it is clear that the growth rate of the deformation of the interface measured in the cross-cut visualizations shown in figure 6 must be related to the growth rate of the instabilities. However, the existence of such a correlation needs to be clearly demonstrated.

To confirm this correlation, we performed a set of temporally evolving simulations using a numerical procedure given in Delbende \& Chomaz (1998). We numerically investigated the two-dimensional linear stability of the Bickley jet with a unit velocity defect $\Theta$ and a unit thickness $\bar{\delta}$ on a periodic box of fixed length $L$. To mark the interface, we 'injected' at time $t=0$ several lines of passive scalars at different distances from the symmetry axis of the Bickley jet. In order to make comparisons with theoretical results, all the simulations initialized by a random perturbation in the velocity field were performed for $R e=\Theta \bar{\delta} / \nu=10^{3}$. Two sets of computations were investigated. In the first one, the Navier-Stokes equations were linearized, but the velocity perturbations were allowed to reach an order-unity amplitude leading to nontrivial passive scalar dynamics, the passive scalar being advected by the full velocity field (i.e. the base flow added to the perturbation). In the second set, the fully nonlinear evolution of the vorticity was computed and the perturbations in velocity were allowed to saturate. Both sets of simulations gave similar results initially and differed when the nonlinear effects became strong. Since we measure experimentally the growth 


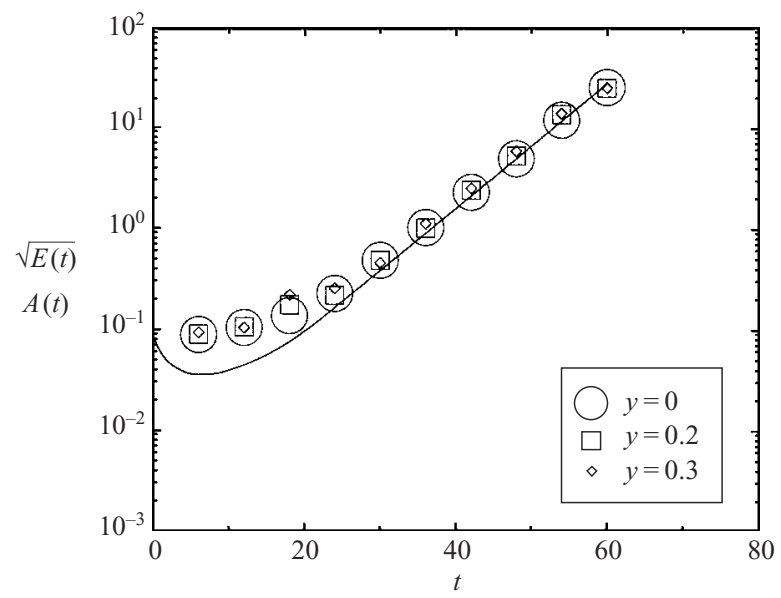

FiguRE 7. Time evolution of the square root of the energy $\sqrt{E(t)}$ (curve) and maximum amplitude deformation of the two-dimensional Bickley jet instability (symbols). The Bickley jet is defined as $U=1-\operatorname{sech}^{2}(y)$, the velocity defect $\Theta$ and the Bickley wake thickness $\bar{\delta}$ being equal to 1 .

rate of the two-dimensional and three-dimensional instabilities during their initial evolution, here we will present an example corresponding to the first set. Figure 7 shows the computed evolution in time of both the square root of the energy $\sqrt{E(t)}$ and the amplitude of the interface deformation $A(t)=\left(A_{\max }(t)-A_{\min }(t)\right)$ for different locations $y=0, y=0.2$ and $y=0.3$. Since we have imposed a two-dimensional wavelength $(\lambda=2 \pi / L)$, and we have initialized the simulations by a white noise, all the harmonics were initially excited: $\lambda=2 n \pi / L, n$ being an integer. In the present case, all but one for $n=1$ were dampened during the initial stage of the simulations resulting in the initial decrease of the energy (from $t=0$ to $t=20$ ), figure 7 . After this transient, $\sqrt{E(t)}$ grows exponentially. Note that at this stage the interface deformation amplitude $A(t)$ also starts to grow exponentially with the same growth rate, whether the offset in $y$ is zero or not. Figure 7 shows, therefore, the excellent agreement between the growth rate measured from the development of the vorticity perturbation field and the growth rate of the interface deformation even when the interface is not located exactly at the symmetry plane. These growth rates computed from the deformations of the interface are also in good agreement with the theoretical inviscid calculation of Drazin \& Howard (1966) since $R e=10^{3}$ is large enough for the viscous damping to be nearly negligible. In particular, in the worst case, when the interface is located in $y=0.3$, Julien (2000) has shown that the error in the growth rate is less than $5 \%$ when the wavenumber varies over the whole unstable domain. The above relationship between interface deformation and growth rate of the instability should apply to three-dimensional perturbations since Lasheras \& Meiburg (1990) have compared three-dimensional deformations of a passive scalar sheet to deformations of the vorticity layer and found that the early growth of the instability (i.e. when three-dimensional perturbations are small) can be evaluated by measuring the spanwise deformations of the passive scalar sheet. These results may be understood by considering the dynamics of vorticity. In the present experiment and numerical simulation, the limit of the dyed layer marks initially a vorticity surface (i.e. at each point of this surface the vorticity vector is parallel to the surface). If we neglect viscosity for the free evolution of the wake, Kelvin's theorem tells us that this 


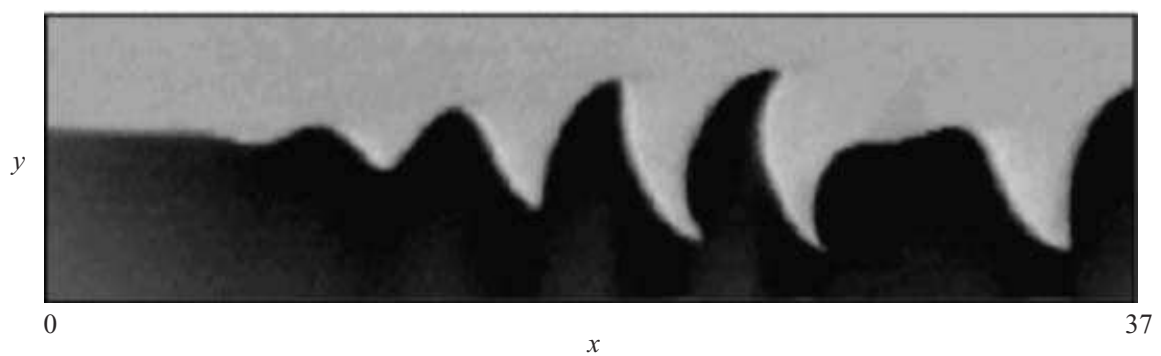

Figure 8. Longitudinal visualization of the unforced Kármán vortex street, $R e_{1}=220$, $z=z_{c}$ near the centre of the channel. Scales are identical in $x$ and $y$.

surface of vorticity evolves as a material surface. The initial correlation between the passive scalar (the dye) and the vorticity is therefore preserved during their evolution. In particular, if the dyed interface coincides initially with the surface of zero vorticity (perfectly symmetric case), then for all times it will mark the location where the vorticity vanishes. If the spanwise vorticity does not vanish at the limit of the dyed layer (non-symmetric case), the orientation of the vorticity and its magnitude will evolve but its direction will stay parallel to the interface. The roll-up of the interface coincides therefore with the roll-up of vorticity. The above argument supports our assumption that monitoring the deformation of the interface provides information about the dynamics of the vorticity in the flow. When the dye injection is not correlated with the vorticity injection in the flow, this relationship does not hold. In the classical work of Hama (1962), the passive scalar was continuously introduced at a fixed crosswise distance in a steady flow periodic in the streamwise direction (here, the hyperbolic tangent velocity profile perturbed by the linear neutral eigenmode with a finite amplitude). Over time, the dye was emitted on different vorticity surfaces, and, as Hama showed, its evolution was then not related to vorticity surface deformation. If, however, he had followed a vorticity surface with the dye emission, he would have observed a steady pattern characteristic of the steadiness of the flow.

In this paper, we will use the above demonstrated correlation between the growth rate of the instability and the growth rate of the amplitude of the interface deformation to measure the growth rate of the instabilities.

\section{Evolution of the primary two-dimensional instability under unforced and forced conditions}

The PLIF visualization of the longitudinal sections (figure $6 a$ ) was used to study and characterize the primary two-dimensional instability. When the wake develops naturally under the inherent low-level background noise of the experimental set-up, the primary instability grows slowly in space and saturates far downstream, forming an irregular Kármán vortex street. The irregularity is quite apparent in the visualization shown in figure 8 , where the local wavelength is considerably larger on the right-hand side of the frame than on the left.

To characterize the temporal irregularities in the wavelength of the two-dimensional mode, we analysed the temporal evolution of the interface at each downstream location $x$. This can be easily obtained from the longitudinal visualizations by combining the temporal evolution of a single column in the digitized sequence of images as shown in figure 9. In all the experiments, the images were acquired at 30 f.p.s. with very high shutter speeds $(45 / 10000 \mathrm{~s})$. Each, temporal diagram in 
(a)

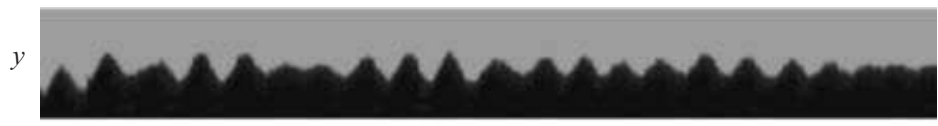

(b)

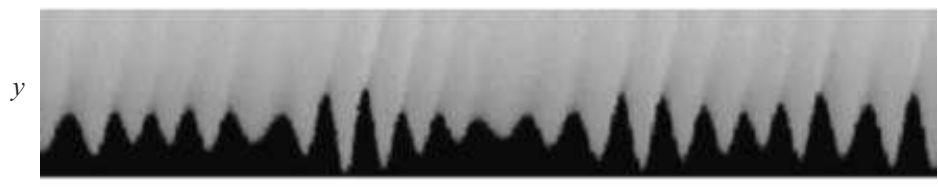

(c)

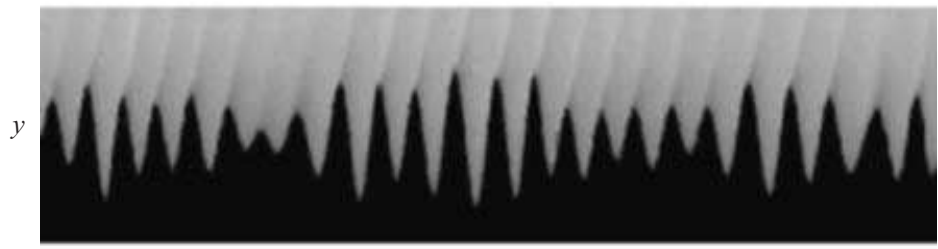

$(d)$


FIGURE 9. Temporal reconstitution of the unforced two-dimensional Kármán vortex street, $R e_{1}=220, z=z_{c}$ near the centre of the channel. $(a) x=3 \mathrm{~cm},(b) x=7 \mathrm{~cm},(c) x=11 \mathrm{~cm}$, (d) $x=18 \mathrm{~cm}$.

figure 9 was taken at a different streamwise location: $x=3 \mathrm{~cm}, x=7 \mathrm{~cm}, x=11 \mathrm{~cm}$ and $x=18 \mathrm{~cm}$. Observe that at each downstream location, the time evolution of the interface is roughly sinusoidal with a constant period. The amplitude was also found to be irregular with a mean value which increases with the downstream distance.

To characterize the two-dimensional mode of the wakes, we calculated the Strouhal number, $S t=(\bar{\delta} f / \bar{U})$, defined by the mean value of the two-dimensional frequency of the vortex street, $f$, the characteristic length, $\bar{\delta}$ (which is the width of the wake at the trailing edge of the splitter plate defined in $\S 3$ ), and the mean velocity of the free stream, $\bar{U}$. The mean frequencies of the naturally evolving wakes were measured to be $f_{1}=1.2 \mathrm{~Hz}$ and $f_{2}=1 \mathrm{~Hz}$, which correspond to a constant Strouhal number of 0.07 for both cases, $R e_{1}=220$ and $R e_{2}=200$. This Strouhal number is in good agreement with the linear stability theory of the Bickley wake (Drazin \& Reid 1981, p. 234). 


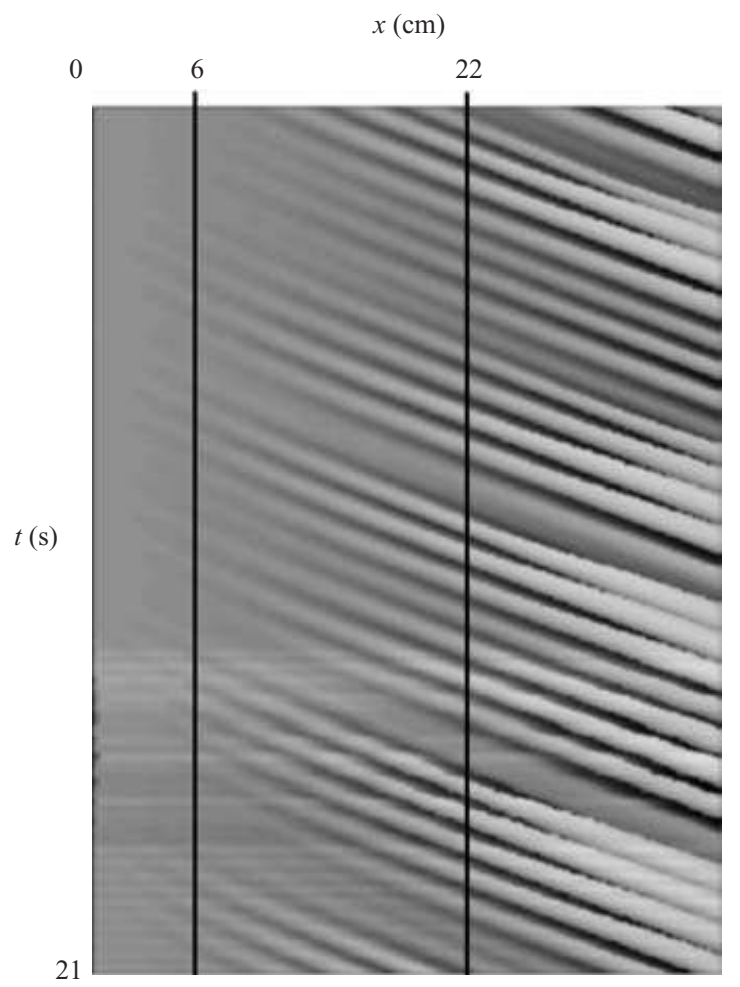

FIGURE 10. Two-dimensional spatio-temporal diagram of the interface height for the unforced wake, $R e_{1}=220, z=z_{c}$ near the centre of the channel.

Indeed, the maximum amplification of the even mode is obtained at the wavenumber $k=1$, which corresponds to a Strouhal number of about $S t=(k V / 2 \pi) \bar{\delta} / \bar{U}=0.06$, when the phase velocity is assumed equal to $0.85 \bar{U}$ as measured in the present experiment (see figure 10 and its discussion hereafter).

To measure the local wavelength of the primary instability, we monitored the deformation of the interface both in time and in the streamwise direction at the centre of the channel, $z_{c}$.

Figure 10 shows the value of the interface displacement $h\left(x, z_{c}, t\right)$, plotted in grey scale, as a function of both time and the streamwise coordinate, $x$. In this case, we observed the appearance of waves, clearly observable from $x=6 \mathrm{~cm}$ onward. The waves propagate downstream while increasing in magnitude. The primary instability is randomly varying around a well-defined mean wavelength. Therefore, its wavelength may be determined from the frequency by using a phase velocity which is independent of $x$. In the $(x, t)$ diagram of figure 10 , the phase velocity, $V$, is determined by the inverse of the slope of the oblique stripes. This slope is much more regular than the wavelength, showing that the phase speed varies little with the wavenumber $k$. This phase speed increases slightly (about $10 \%$ ) as the primary instability saturates. We found asymptotic phase speeds $V=5.5 \mathrm{~cm} \mathrm{~s}^{-1}$ and $V=4.6 \mathrm{~cm} \mathrm{~s}^{-1}$, for $R e_{1}=220$ and $R e_{2}=200$, respectively, which correspond to $V=0.85 \bar{U}$ in both cases.

To force the primary instability leading to the formation of a regular Kármán vortex street, we used the forcing technique described in $\S 3$ and previously used by Schowalter et al. (1994). As one would expect, although the forcing frequency 


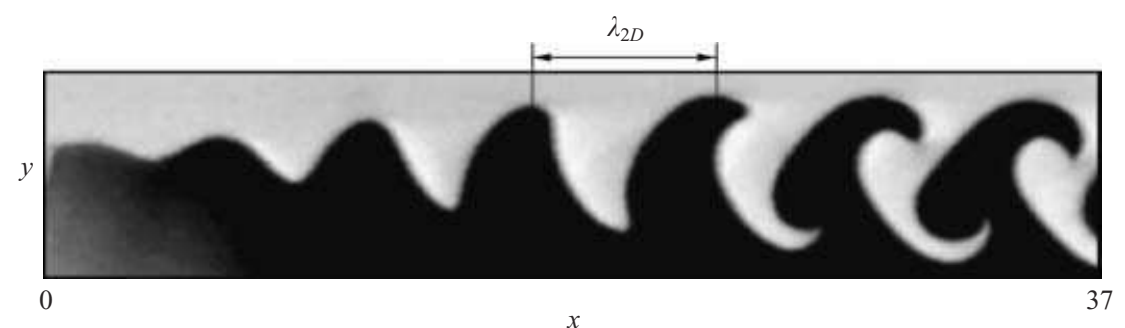

FIGURE 11. Longitudinal visualization of the forced Kármán vortex street, $R e_{1}=220$, $S t=0.07, z=z_{c}$ near the centre of the channel. Scales in $x$ and $y$ are identical.

was varied over a large range of Strouhal numbers $S t$, from 0.04 to 0.16 , a regular Kármán vortex street was obtained for a subrange of forcing frequencies only. In the case of $R e_{1}=220$, the Kármán vortex street was found to be regular in space (figure 11) and time (figure 12) for Strouhal numbers ranging from 0.06 to 0.09 . Since the forcing amplitude is larger than the amplitude of the background noise, the amplitude of the primary instability measured in these cases was always found to be larger than in the natural case, forming a more regular vortex pattern (compare the natural case figures 8, 9 and 10 and the forced case figures 11, 12 and 13). Note that in the forced case the interface has nearly overturned at the downstream location $x=11 \mathrm{~cm}$, figure 12, whereas in the natural case it is still developing (figure 9). Phase velocities, $V$, evaluated on spatio-temporal diagrams such as in figure 13, reach their asymptotic value sooner. For all the forcing frequencies tested, the phase velocity $V$ is always found to be equal to $0.85 \bar{U}$.

\section{Three-dimensional vorticity patterns in the wake of a flat plate}

\subsection{The near wake}

In this section, we consider the initial evolution of the wake in the first 2 to 3 wavelengths downstream of the splitter plate (to about $x=11 \mathrm{~cm}$ ). This region will be referred to as the near wake. Both naturally developing (unforced) and forced cases will be discussed here. In these early stages, since there is no folding of the interface, the measurement of the parameter $h(x, z, t)$, the height of the interface measured from the top of the frames, is sufficient to reconstruct the entire evolution of the interface.

\subsubsection{Unforced case}

Despite the irregularity in the frequency and amplitude of the primary instability obtained in the natural unforced case, the secondary three-dimensional instability was always observed to develop, producing deformations of the interface in the spanwise direction. In order to identify the dominant mode in this region, we conducted a series of visualizations aimed at identifying each of the modes by detecting the symmetry characteristics discussed in $\S 2$. Since one distinct feature of these modes is the appearance of either a sinusoidal (in-phase) undulation or a varicose (outof-phase) undulation in the end views of the Kármán vortices, we super-imposed 20 consecutive end-view PLIF images (the frame rate in this case was 15 f.p.s.). Figure 14 shows a representative experiment in the naturally evolving unforced case. It shows the concentration of dye averaged during one and one half periods of the primary instability. In this averaged visualization, one can see the deformation of 
(a)

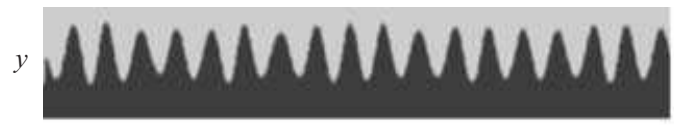

(b)

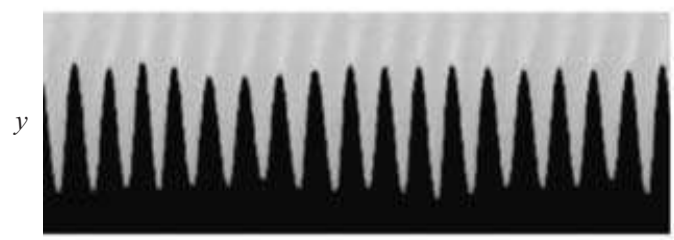

(c)

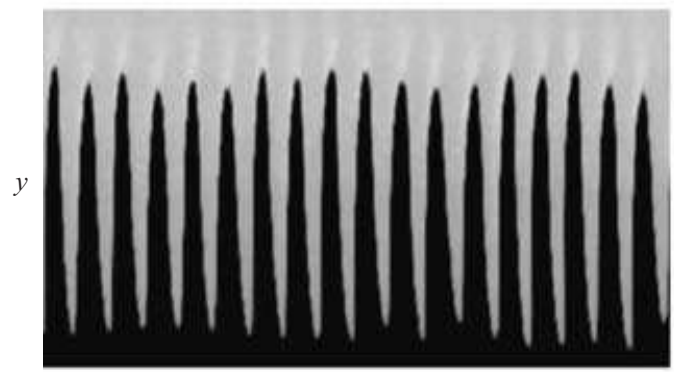

(d)

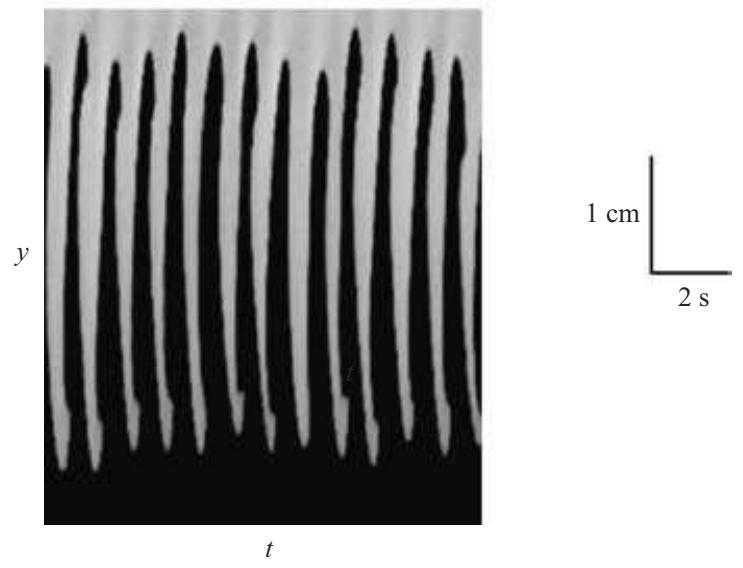

Figure 12. Temporal reconstitution of the forced two-dimensional Kármán vortex street, $R e_{1}=220, S t=0.07, z=z_{c}$ near the centre of the channel. (a) $x=3 \mathrm{~cm},(b) x=7 \mathrm{~cm}$, (c) $x=11 \mathrm{~cm},(d) x=18 \mathrm{~cm}$.

the interface resulting from both the primary and the secondary three-dimensional instabilities. The symmetry characteristics of the mean concentration in the $(y, z)$ plane (figure 14) reveal a varicose shape, indicating that the selected three-dimensional mode was Mode 2 (compare this figure with the vortex dynamic simulations shown in figure $2(b)$. However, in the unforced experiments, where the naturally developing primary instability is irregular, we found that the secondary instability, as characterized by its wavelength, was also irregular and unsteady. The unsteadiness and irregularities 


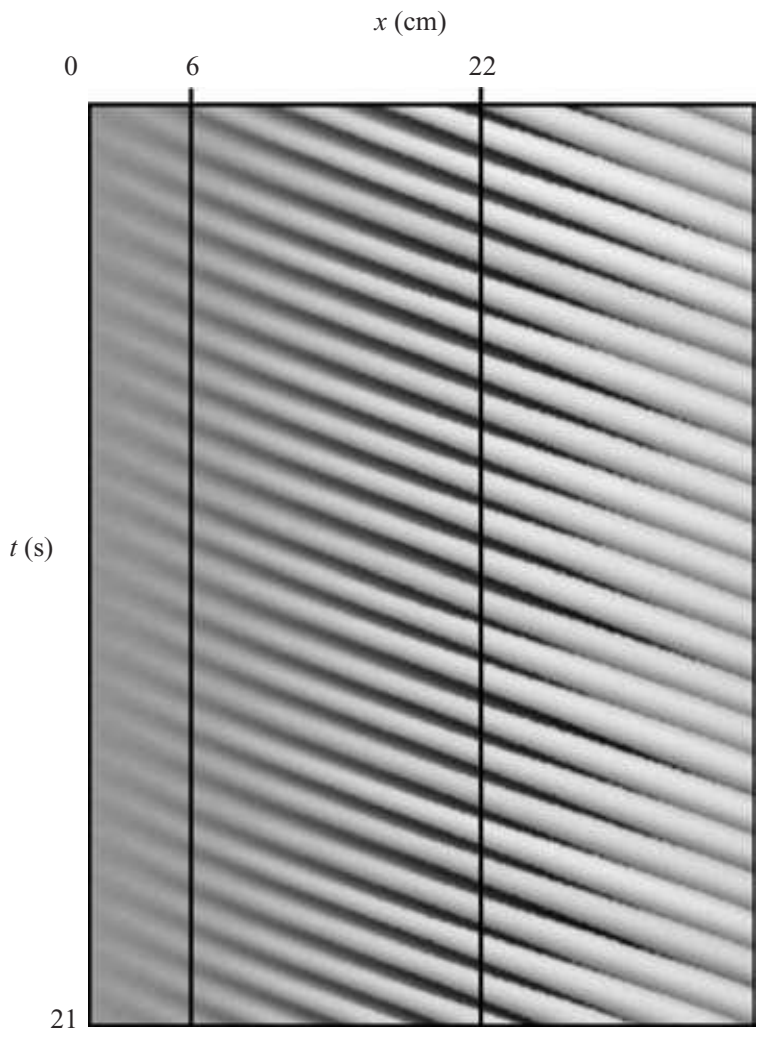

FIGURE 13. Two-dimensional spatio-temporal diagram of the interface height for the forced wake, $R e_{1}=220, S t=0.07, z=z_{c}$ near the centre of the channel.

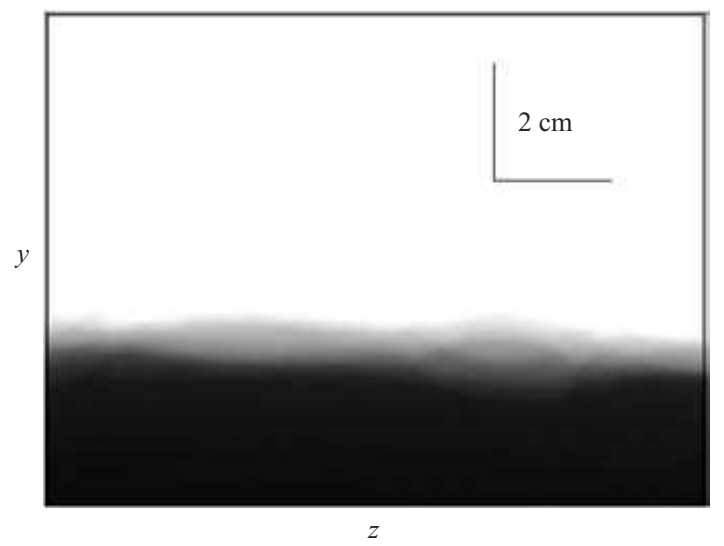

Figure 14. Mode 2, $R e_{1}=220, x=11 \mathrm{~cm}$.

in the spanwise wavelength of the three-dimensional instability can be observed in the spatio-temporal diagram of the interfacial parameter $h(x, z, t)$ (figure 15).

The representation of the value of this interface parameter as grey levels is similar to those shown in figures 10 and 13 except that now we use the visualization of the spanwise end views instead of the side views to measure $h(x, z, t)$. Using the 




FIGURE 15. Spatio-temporal diagram of the irregular Kármán vortex street, unsteady Mode 2, $R e_{1}=220, x=11 \mathrm{~cm}$. The spanwise length shown (z-axis) is $11 \mathrm{~cm}$.

Taylor hypothesis, assuming that all the structures are advected at a constant phase velocity $V=0.85 \bar{U}$, the time evolution depicted on figure 15 may be transformed into a streamwise evolution. A spatio-temporal diagram such as figure 15 allows us to compare directly the streamwise deformations due to the primary instability to the spanwise deformations due to the secondary instability. In order to represent long time sequences, we applied a factor of two between the spanwise coordinate and the equivalent streamwise length scale obtained by multiplying the time by the phase velocity $V$ of the two-dimensional structure. The primary instability, which induces the time-periodic oscillations of the interface in a cross-stream section, appears as a spanwise stripe (i.e. a vertical stripe on figure 15) since the darker the pixel the lower the interface location. Thus, the irregularity in the frequency of the two-dimensional Kármán vortex street appears as the varying distance between the spanwise stripes, and the irregularity in its amplitude appears as a variation along the span of the intensity of the white and dark stripes (the darker and whiter stripes indicate the larger interface deformation associated with the larger amplitude of the two-dimensional mode). The appearance of the three-dimensional instability is manifested by a waviness of the vertical lines, corresponding to the streamwise distortion of the primary spanwise vortices. The streamwise distortion of the Kármán vortices which remains in-phase during the streamwise evolution is a characteristic feature of Mode 2 (see figures $1 c, d$ and $3 b$ ). Mode 2 induces an 'out-of-phase' undulation of two consecutive spanwise vortices in a vertical $(y, z)$-plane that translates into a variation in the amplitude of the interface displacement along the span. This modulation of the amplitude of the undulation corresponds in the spatio-temporal diagram to a variation in the contrast of the black and white stripes in the spanwise direction ( $z$-axis) as can be seen in figure 15 .

To confirm further that we have observed the appearance of Mode 2 in this unforced case, despite the irregularity of the two-dimensional and three-dimensional instabilities, we analysed the evolution of the interface over a single period of the primary instability. This was done by super-imposing the location of the interface measured every one tenth of the period on the same diagram (figure 16). Note that in the first half-period (shown in black) the deformations of the interface along the span are approximately symmetric with the deformations of the interface during the second half (shown in white). This behaviour corresponds to the varicose shape characteristic of Mode 2 (see figure $2 b$ ).

\subsubsection{Two-dimensional forcing case}

Under the effect of two-dimensional forcing, described in $\S 3$, the three-dimensional instability always develops naturally (see figures 17,18 and 19). In the near region, Mode 2 continues to appear as the only clearly distinguishable three-dimensional mode, regardless of the two-dimensional forcing frequencies applied. As in the unforced case, we also observed situations where no identifiable three-dimensional 


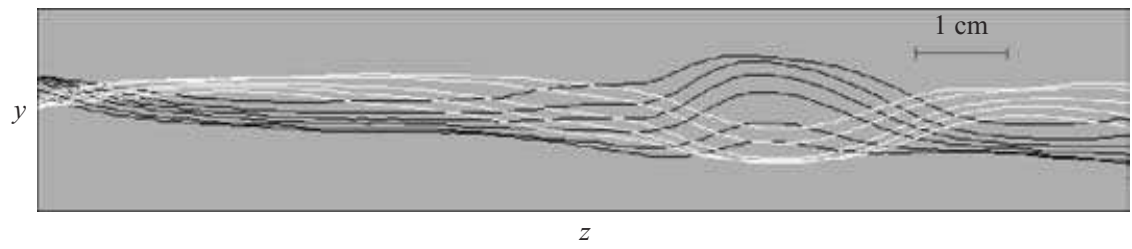

FIGURE 16. Superposition diagram of interfaces during one period of the two-dimensional instability, $R e_{1}=220, x=11 \mathrm{~cm}$. Scales are identical on $y$ and $z$. Black lines are the six interface locations during the first approximate half-period of the two-dimensional instability, and white lines the following five interface locations during the other half-period.

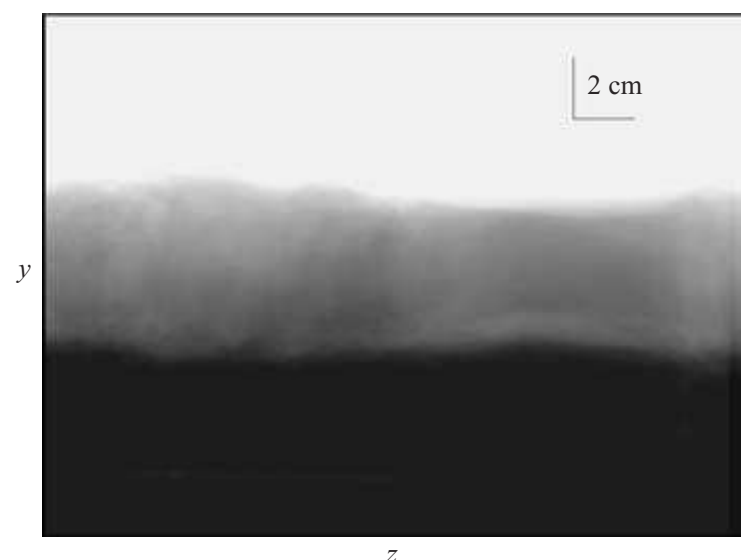

FIGURE 17. Concentration-average image, $R e_{2}=200, S t=0.07, x=10 \mathrm{~cm}$.

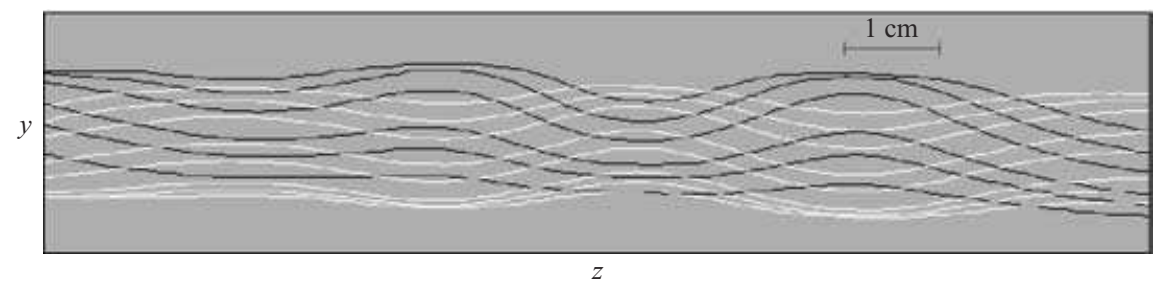

FIGURE 18. Superposition diagram $R e_{1}=220, S t=0.07, x=7 \mathrm{~cm}$.

Scales are identical in $y$ and $z$.

mode was detected. At this stage, we cannot rule out the possibility of the coexistence of both modes creating a complex topology of the vorticity.

Figure 17 shows an image of the averaged concentration (similar to the one shown in figure 14) measured at the downstream section $x=10 \mathrm{~cm}$, for $R e_{2}=200$ with a forcing frequency corresponding to $S t=0.07$, exhibiting the characteristic shape of Mode 2. Likewise, figure 18, which is a superposition diagram for $R e_{1}=220$ and $S t=0.07$, similar to the one shown in figure 16 , also displays the varicose undulation of the interface for each half period.

In the spatio-temporal diagram, figure 19, one can also observe the spanwise modulation of the amplitude of the interface height $h(x, z, t)$ which is visualized by the spanwise variations in the intensity of the periodic black and white stripes. For example, at the spanwise location $\mathrm{A}$, the amplitude of the modulation is smaller than 
(a)

$R e$

200

220

\begin{tabular}{l}
\multicolumn{1}{c}{$S t$} \\
0.07 \\
0.084 \\
0.06 \\
0.07 \\
0.08 \\
0.09
\end{tabular}

,

$\frac{\lambda_{3}}{\lambda_{2}}$
1
1

0.9

0.95

1.05

0.9

\begin{tabular}{ccc} 
& $(b)$ & \\
\hline$S t$ & $\frac{\lambda_{3 D}}{\lambda_{2 D}}$ & $\frac{\lambda_{3 D}}{\delta}$ \\
0.07 & 1 & 12 \\
0.06 & 1 & 14
\end{tabular}

10

13

12

11

9

TABLE 1. Ratios $\left(\lambda_{3 D} / \lambda_{2 D}\right)$ and $\left(\lambda_{3 D} / \bar{\delta}\right)$ for different $S t$ and both Reynolds numbers. $(a)$ three-dimensional forced cases, $(b)$ three-dimensional natural cases. A round off has been applied to take into account the error due to the variability of $\lambda_{3 D}$ in $(a)$ and $\lambda_{2 D}$ in $(b)$.

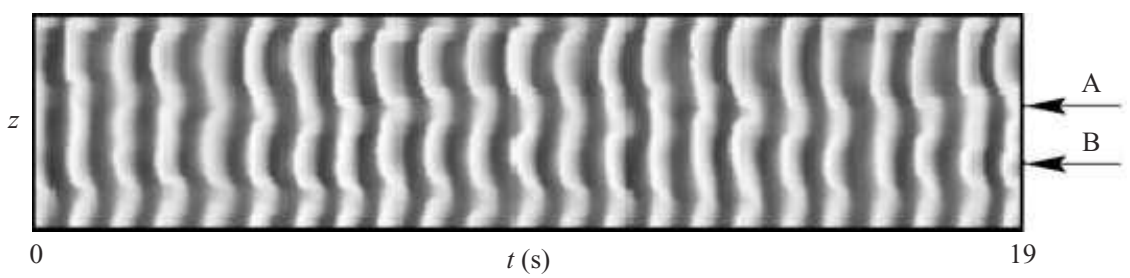

FIGURE 19. Spatio-temporal diagram, $R e_{1}=220, S t=0.07, x=11 \mathrm{~cm}$. The spanwise length shown (z-axis) is about $11 \mathrm{~cm}$.

at $\mathrm{B}$ and as a result on figure 19 only pale black and blurred grey are visible at the $z$-location A whereas black and white are clearer on the line B. On the other hand, in this figure one can also observe the 'in-phase' longitudinal distortion of the crest and trough of the interface characteristic of the streamwise undulation of the Kármán vortices shown in figure $3(b)$, confirming the appearance of Mode 2. It is important to point out that this spatio-temporal diagram also demonstrates that the secondary instability appears to be not stationary, i.e. the amplitude modulation and waviness are seen to move slowly along the span with a spanwise wavelength that varies around a well-defined mean value. Measurements similar to figure 19, made every $2 \mathrm{~cm}$ at increasing downstream locations (not presented here), show that the mean wavelength of Mode 2 remains reasonably constant. Furthermore, by varying the forcing Strouhal number of the primary instability, we observed that the threedimensional wavelength, $\lambda_{3 D}$, of Mode 2 is always of the same order of magnitude as the wavelength of the two-dimensional mode, $\lambda_{2 D}$. The two-dimensional wavelength is estimated as the ratio $(V / f)$, where $V$ is the phase velocity defined previously and $f$ is the applied forcing frequency of the primary instability. The mean three-dimensional wavelength was measured in the concentration-averaged images (see figure 17), in the superposition diagrams like the one shown in figure 18 and in the spatio-temporal diagrams of figure 19 , always obtaining identical results.

Table 1 shows the measured ratio $\left(\lambda_{3 D} / \lambda_{2 D}\right)$ as a function of the Strouhal number $S t=(f \bar{\delta}) / \bar{U}$. As we varied the velocity and the two-dimensional forcing frequencies, the ratio $\left(\lambda_{3 D} / \lambda_{2 D}\right)$ was always found to be around 1 . Thus, we concluded that the wavelengths of the primary and the secondary instability were of comparable magnitude. The values of $\left(\lambda_{3 D} / \bar{\delta}\right)$ are also shown in table 1 . Note that they vary from 
(a)

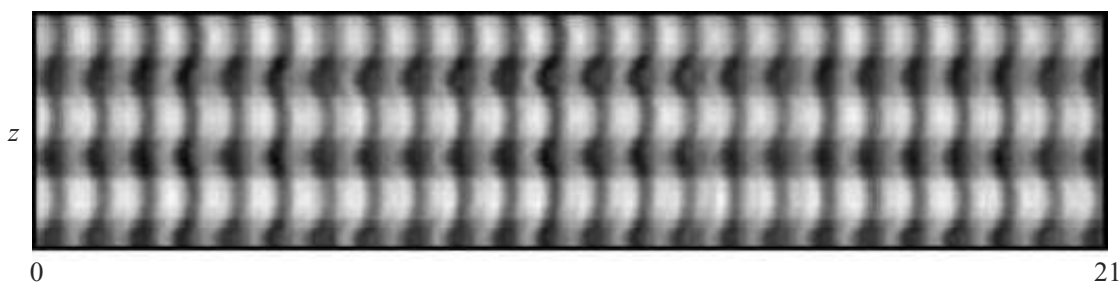

(b)

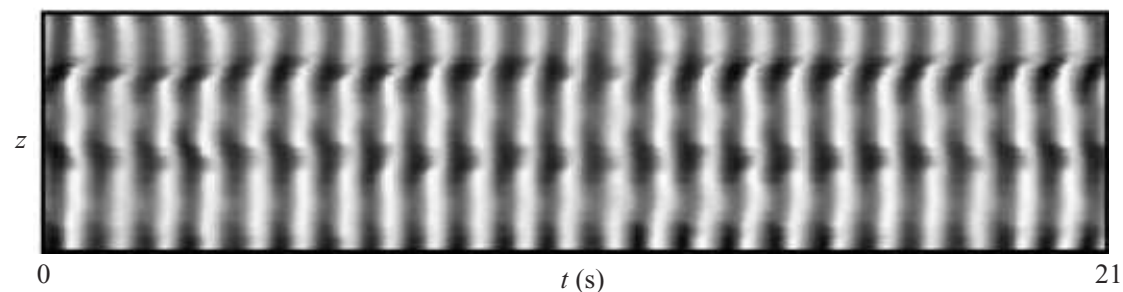

FiguRE 20. Spatio-temporal diagram, $R e_{1}=220, S t=0.07$. Adjustment to a preferred threedimensional wavelength, $\lambda_{3 D} / \lambda_{2 D}=0.76,(a) x=1 \mathrm{~cm},(b) x=7 \mathrm{~cm}$. The spanwise length shown ( $z$-axis) is $11 \mathrm{~cm}$. The grey scale has been adjusted to maximize the contrast in the figure only, not in the analysed field.

9 to 14 , demonstrating that the selection of the wavelength of the secondary instability is apparently based on the wavelength of the primary vortices and not on the width of the initial wake profile. This result is consistent with Pierrehumbert \& Widnall's (1982) translative instability computation for the mixing layer, and Brancher's (1996) stability analysis of jets which show that the wavelength of the secondary instability is mainly determined by the wavelength of the two-dimensional primary instability.

\subsubsection{Two- and three-dimensional forcing case}

In order to investigate quantitatively the selection of the wavelength of the secondary instabilities, we also performed an additional series of experiments by forcing Mode 2 at varying wavelengths using indented trailing edges of the splitter plate. This technique, introduced and discussed in detail by Meiburg \& Lasheras (1988), was shown to result in Mode 2. To vary the wavelength of the spanwise perturbation (the wavelength of the three-dimensional mode), we used 10 different shapes of trailing edges with wavelengths varying from $2.5 \mathrm{~cm}$ to $6 \mathrm{~cm}$. The amplitude of the indentation was kept constant in all the cases and equal to $1 \mathrm{~cm}$.

Figures 20 and 21 show the spatio-temporal evolution of the wake forced at $S t=0.07$ for two different three-dimensional forcing wavelengths. In both cases, the flow was found to develop a Mode 2 with the imposed wavelength. Consistent with the development of this mode, we observed the spanwise modulation of the contrast, and the 'in-phase' undulation between black and white stripes. The regularity in the spatial and temporal evolution of these undulations indicates the high receptivity of this mode to the applied three-dimensional forcing. However, we also observed that when the forcing wavelength was increased to a value much larger than the one measured that appeared naturally (i.e. $\lambda_{3 D}$ too large compared to $\lambda_{2 D}$ ), the receptivity to this forcing was weak and the three-dimensional pattern became highly irregular (figure $21 b$ ). On the other hand, when the imposed three-dimensional forcing 
(a)

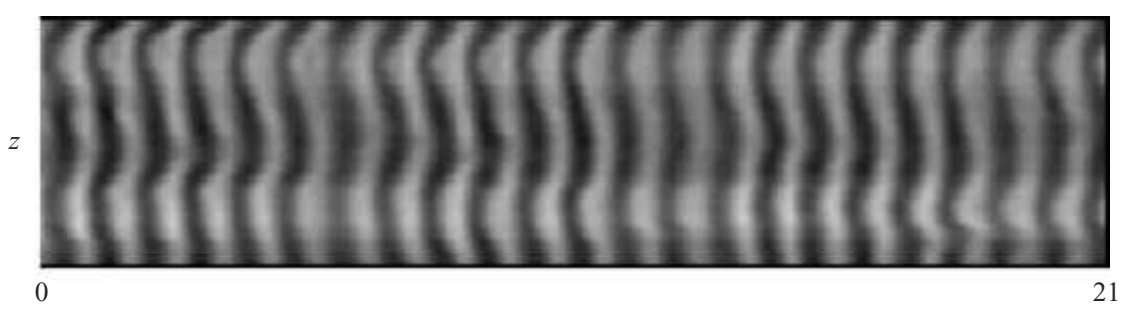

(b)

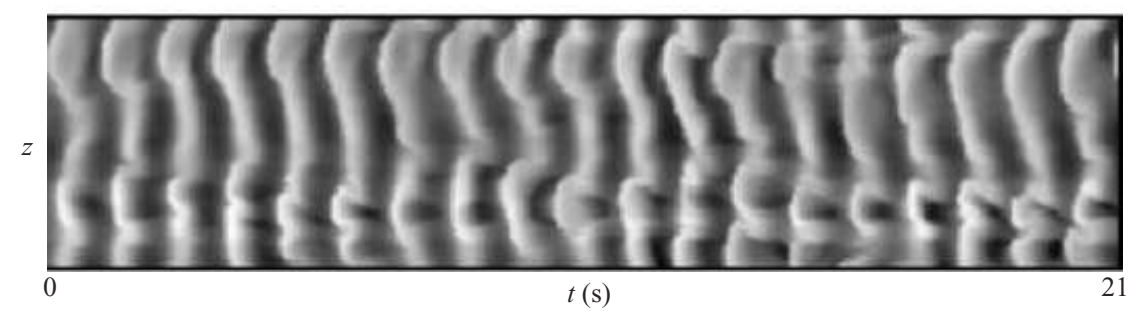

FIgURE 21. Spatio-temporal diagram, $R e_{1}=220, S t=0.07, \lambda_{3 D} / \lambda_{2 D}=1.29$. The threedimensional wavelength imposed is not followed, $(a) x=1 \mathrm{~cm},(b) x=8 \mathrm{~cm}$. The spanwise length shown (z-axis) is $11 \mathrm{~cm}$.

wavelength was close to the natural one (i.e. $\lambda_{3 D} \approx \lambda_{2 D}$ ), we found that the receptivity was very high, and the growth of the imposed mode dominated the entire evolution of the flow (figure $20 a, b$ ).

The spatio-temporal diagrams corresponding to these forced experiments contain a large amount of information on the characteristic features of the two-dimensional and three-dimensional flow field, i.e. the interface oscillations in time due to the presence of the staggered Kármán vortices array, the spanwise amplitude modulation of the interface deformation, and the in-phase undulations in the streamwise direction of the Kármán vortices caused by the development of Mode 2. To study these important features of the secondary instability, we isolated its phase and amplitude from the oscillating $h(x, z, t)$ signal by applying a two-dimensional Fourier transform to the spatio-temporal diagram, figure $20(b)$. This allowed us to obtain the complex twodimensional spectrum, which gives the fundamental and all the harmonic frequency peaks, characteristic of the interface oscillations in time and in space. In addition, we filtered it around the fundamental frequency in order to recover only the spanwise modulations of the fundamental oscillations. By keeping half of the filtered frequency spectrum (i.e. setting to zero the amplitude of the Fourier component) and by applying an inverse complex-to-complex Fourier transform, we changed the real initial signal into a complex signal. This procedure is equivalent to performing a Hilbert transform on the real signal. The phase and the modulus of the complex field obtained correspond to the phase and the amplitude of the modulation of the interface oscillation.

The phase diagram (figure 22a), shows spanwise, wavy, contrasted lines associated with the 'in-phase' distortions of Kármán vortices. The measured amplitudes, figure 22(b), show a well-organized array of streamwise stripes, a characteristic of the spanwise amplitude modulation of the interface deformation of Mode 2 (black 
(a)

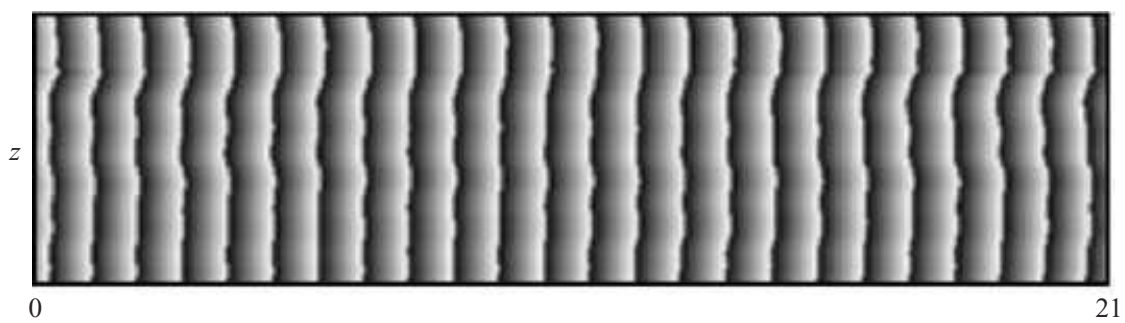

(b)

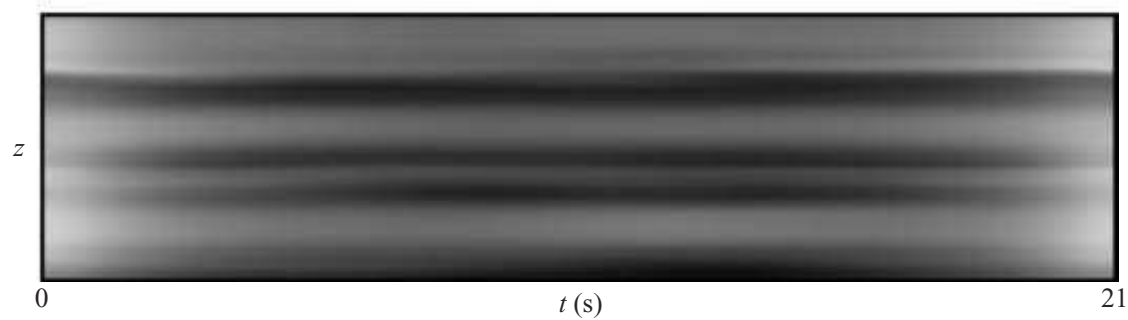

FiguRE 22. Demodulation of the spatio-temporal diagram $R e_{1}=220, \quad S t=0.07$, $\lambda_{3 D} / \lambda_{2 D}=0.76$ : $(a)$ phase diagram, $(b)$ amplitude diagram. The spanwise length shown $(z$-axis) is about $11 \mathrm{~cm}$.

corresponding to large amplitudes and grey to low). The time sequence was chosen to be long enough for the end effect of the demodulation to be weak.

As we followed the evolution of the interface between the two streams, the growth of the amplitude of the secondary instability in the early development of three-dimensionality provided us with a measurement of the growth rate of the three-dimensional instability which allowed us to confirm the selection of the secondary instability wavelength. We defined the amplitude of the secondary instability as the spanwise modulation of the time oscillations of the interface. This can be measured in the averaged pictures (figure 17) of the mean concentration of dye, by fixing the upper intensity threshold at $95 \%$ of the maximum pixel intensity and the lower threshold at $5 \%$ of the maximum intensity. Note that similar results are obtained if the amplitude of Mode 2 is defined by the difference between the maximum and the minimum of intensity measured on the demodulated amplitude diagrams (figure $22 b$ ). In figure 23(a), we plot the measured amplitudes as a function of the streamwise distance, $x$. One of the cases shown corresponds to conditions where the secondary instability was not forced, whereas in the three other cases, three-dimensional forcing was applied at three different spanwise wavelengths. For clarity in the presentation, we have not plotted all the three-dimensional forcings tested since they show an identical trend. Note that the perturbation corresponding to $\lambda_{3 D} / \lambda_{2 D}=0.76$ (triangles in figure $23 a$ ) grows much faster than all the others. In this case, the amplitude of the three-dimensional wave measured at $x=3 \mathrm{~cm}$ was found to be two times larger than in any other case. The three-dimensional growth rate was calculated by the initial slope of the amplitude curves (figure $23 \mathrm{~b}$ ). For $\lambda_{3 D} / \lambda_{2 D}=0.59$, the growth is slow enough that the first four points are aligned on the linear-log plot figure $23(a)$ and 

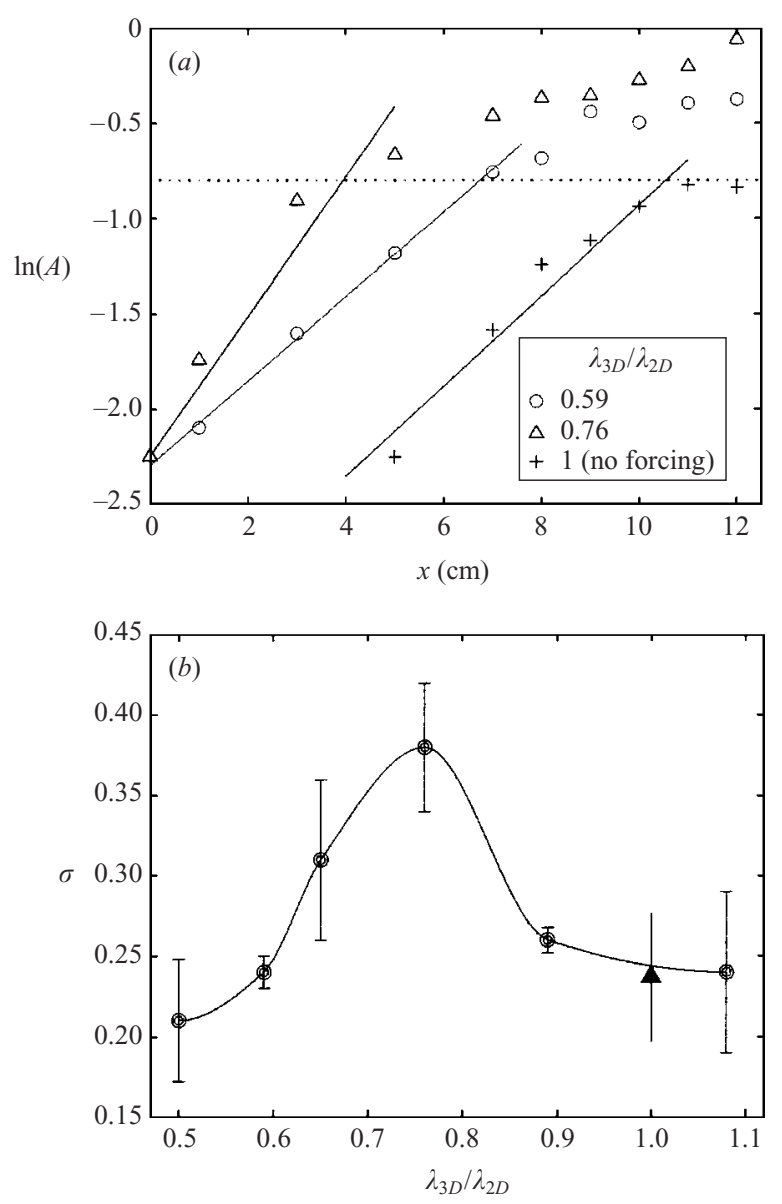

FIGURE 23. Selection of a wavelength: (a) downstream evolution of the amplitude of Mode 2, $A_{3 D}(x),(b)$ growth rate, $\sigma\left(\mathrm{cm}^{-1}\right)$ of the three-dimensional instability measured from the initial slope of curves in $(a)$. Crosses correspond to the unforced case.

around $\ln (A)=-0.8$ the amplitude of the three-dimensional deformations starts to be comparable to the amplitude of the two-dimensional deformations, meaning that the secondary instability has reached an order-unity amplitude. For all the forcing wavelengths, this value indicated by a dotted line on figure 23(a) marks a decrease in the growth rate of the interface deformation and the beginning of the nonlinear regime. Although for $\lambda_{3 D} / \lambda_{2 D}=0.59$, an initial growth rate may be computed accurately, this is not the case for $\lambda_{3 D} / \lambda_{2 D}=0.76$ since the growth is so fast that the nonlinear regime has already been reached at the third point. To estimate the growth rate we have, therefore, only two points that are obviously aligned. To estimate the error of this measurement, we use the fact that all the amplitude curves (shown on figure $23 a$ but not shown for $\lambda_{3 D} / \lambda_{2 D}=0.44,0.59,0.89$ and 1.08) cross approximately at $x=0$ at $\ln (A)=-2.25$. This value may therefore be considered as the initial perturbation amplitude (i.e. at $x=0$ ) imposed by the forcing device. The error in the growth rate for $\lambda_{3 D} / \lambda_{2 D}=0.76$ has been computed by estimating the growth rate at the three points $x=0, x=1, x=3$ and at the three points $x=1, x=3, x=5$. For the unforced case (crosses on figure $23 a$ ), the growth rate has been estimated 
for all the points below $\ln (A)=-0.8$ at which the amplitude saturates. In that case, the variability between one point and the next is large since the flow continues to evolve during the time we perform the measurements. The corresponding growth rate is marked by a solid triangle in figure $23(b)$ located at the mean value of $\lambda_{3 D} / \lambda_{2 D}$ since $\lambda_{3 D}$ is not forced and varies in time. Even though the error bars are large for some measurements, figure $23(b)$ shows that the most amplified secondary instability wavelength is such that $\lambda_{3 D} / \lambda_{2 D}$ is about 0.8 , the fastest (by a factor 2 at $x=3$ ) growing forced mode being $\lambda_{3 D} / \lambda_{2 D}=0.76$.

It is important to note that $\lambda_{3 D} / \lambda_{2 D}$ measured in the unforced case was close to 1 while the above results show a preferred $\lambda_{3 D} / \lambda_{2 D}=0.8$. We do not have a definite explanation for this discrepancy. It may be due to the irregularities and unsteadiness of the natural case: $\lambda_{3 D}$ represents only an average value. Nevertheless, one can conclude from the above experiments that the preferred wavelength of the threedimensional instability is of the order of magnitude of that of the two-dimensional instability.

The growth rate measured here may be compared with the study of Sutherland \& Peltier (1994) on the secondary instability of a von Kármán street issuing from the nonlinear development of the primary instability of a Bickley wake. Their analysis is temporal and therefore applies also to wakes since wake and jet flows differ only in the definition of the reference frame. At time $t=50$ in the roll-up process, the most unstable mode is Mode 1 but Mode 1 and Mode 2 exhibit similar maximum growth rates ( 0.085 for Mode 1 and 0.071 for Mode 2 in non-dimensional variables) which are reached at the same spanwise wavelength corresponding to $\lambda_{3 D} / \lambda_{2 D}=0.77$. The fact that Mode 1 possess the largest temporal growth rate whereas experimentally Mode 2 dominates, is not significant since the difference in temporal growth rates between Mode 1 and Mode 2 is small. In particular, it may be attributed to the fact that the flow is spatially evolving in the experiment and assumed frozen in the stability analysis of Sutherland \& Peltier. On the other hand the most unstable wavelength measured in the experiment $\lambda_{3 D} / \lambda_{2 D}=0.76$ agrees remarkably well with their computation $\left(\lambda_{3 D} / \lambda_{2 D}=0.77\right)$. Comparison for the growth rate is not straightforward since Sutherland \& Peltier have performed a temporal study and we have measured spatial growth rates. Nevertheless, if we use the Gaster (1964) transformation by anticipating the convective nature of the instability demonstrated below (see $\S 5.3$ ), and if we estimate the group velocity of the three-dimensional perturbation $V_{G}$ by the phase speed of the primary vortex (i.e. $0.85 \mathrm{U}$ ), we obtain $\sigma_{\text {exp }}=\sigma_{\text {temp }} U / V_{G} \delta \sim \sigma_{\text {temp }} / 0.85 \delta=0.3 \mathrm{~cm}^{-1}$, in fair agreement with the largest growth rate measured, $0.370 .04 \mathrm{~cm}^{-1}$.

\subsection{The far wake}

In this section, we turn our attention to the evolution of the wake in the far region defined here as downstream distances from 3 to 5 wavelengths, (i.e. $x \in[12 \mathrm{~cm}$; $22 \mathrm{~cm}]$ ). In this far region, the nonlinear effects become important but the wake is still well structured. When the primary two-dimensional instability is forced whether or not the secondary three-dimensional instability is forced, we observed either the formation of Mode 1 or Mode 2, or the coexistence of both. Mode 1 however was present in most cases.

For example, note in the concentration average shown in figure 24 a clearly visible sinuous undulation of the interface, characteristic of Mode 1 (see figure 2a). However, the superposition diagrams of the interface elevations during a single period obtained from the same sequence (figure 25) show that the curvatures of $h(x, z, t)$ from one 


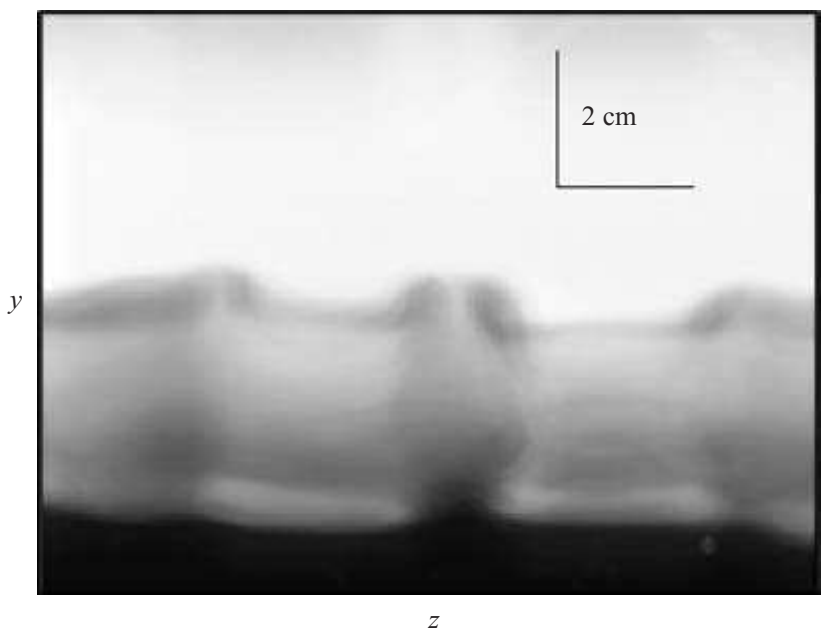

FIgURE 24. Mode $1, R e_{1}=220, S t=0.07, x=18 \mathrm{~cm}$, averaged frames as in figure 14 .

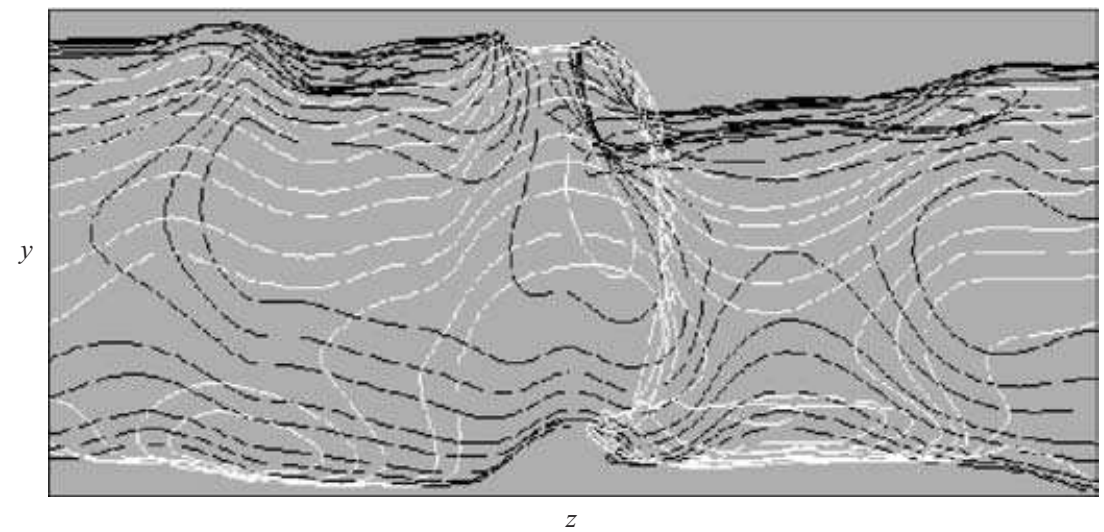

FIgURE 25. Mode $1, R e_{1}=220, S t=0.07, x=18 \mathrm{~cm}$, superposition diagram, as in figure 16 .

half-period (in white) to the next half-period (in black) are opposite in the right part of the graph, whereas at the centre of the graph they are oriented in the same direction. This appears to indicate that Mode 1 is not pure, but coexists with Mode 2 .

In figure 26, we show two series of concentration-averaged pictures presenting the streamwise evolution of the secondary three-dimensional instability. These correspond to two different runs of the experiment using identical conditions. The only difference between the two cases is the level of small random perturbations existing in the initial conditions which, as we explained in $\S 3$, result from an uncontrolled attachment of micro-bubbles (several hundreds of microns in diameter) underneath the splitter plate. In the first series, (case $a$ ), we see that Mode 2 is amplified from $x=10 \mathrm{~cm}$ to the farthest section $x=16 \mathrm{~cm}$; whereas in case $(b)$, Mode 2 dominates the flow until section $x=13 \mathrm{~cm}$, and the sinuous undulation characteristic of the presence of Mode 1 appears at $x=16 \mathrm{~cm}$.

For the far wake considered in this section, $x \in[12 \mathrm{~cm} ; 22 \mathrm{~cm}]$, the interface between the two layers becomes very complex with many foldings and as a result its 

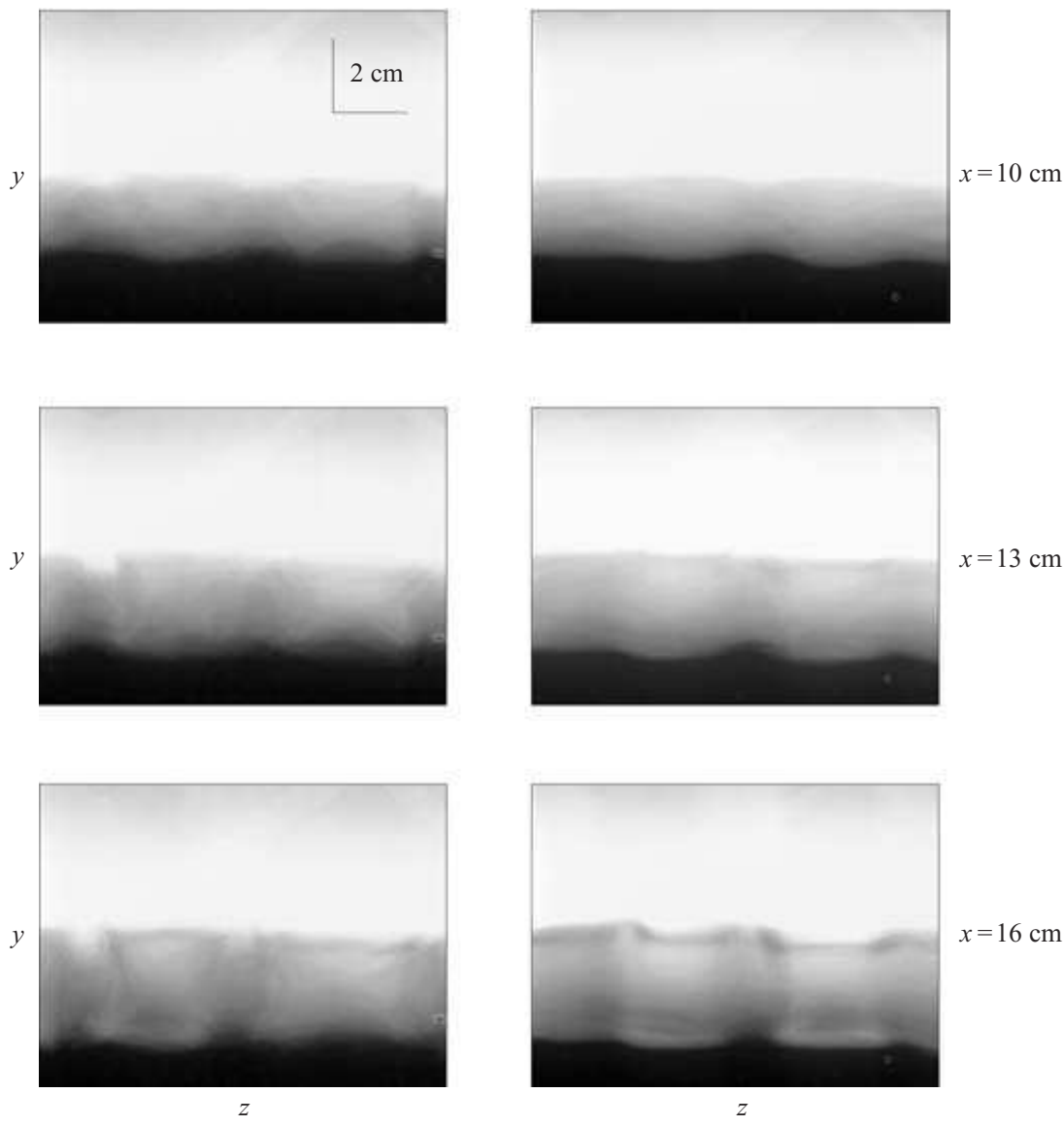

FIGURE 26. Far evolution of the secondary instability, $R e_{1}=220$ : (a) pure Mode 2, (b) Mode 1 contribution.

intersection with a cross-plane is not a simply connected object but rather has the appearance of several lines and closed loops. As we mentioned in $\S 3$, the elevation of the interface $h(x, z, t)$ at a particular location is no longer a single-valued function, and knowledge of the uppermost elevation of the interface (as presented in the spatiotemporal diagrams of figures 20 and 21) is not sufficient to describe the topology of the flow. Nevertheless, we can still describe the interface from the measurements of $h_{u}(x, z, t)$ and $h_{d}(x, z, t)$. To confirm the coexistence of both three-dimensional modes, in figure 27 we plot the time-averaged uppermost $\overline{h_{u}(x, z)}$ and lowermost $\overline{h_{d}(x, z)}$ elevation of the interface. The symmetric and anti-symmetric vertical deformations, characteristic of Modes 1 and 2, respectively, are clearly visible. Note that at the spanwise location marked (1) the sinuous shape characteristic of Mode 1 is quite apparent. However, observe that at the spanwise location (2), a varicose shape characteristic of Mode 2 is clearly shown.

The development of Mode 1 in this far region could be due to the amplification of external noise at a growth rate smaller than that of Mode 2, or may be due to the linear transfer between modes generated by the eventual small asymmetry of the base velocity profile. The seemingly random appearance of Mode 1 and its coexistence with 


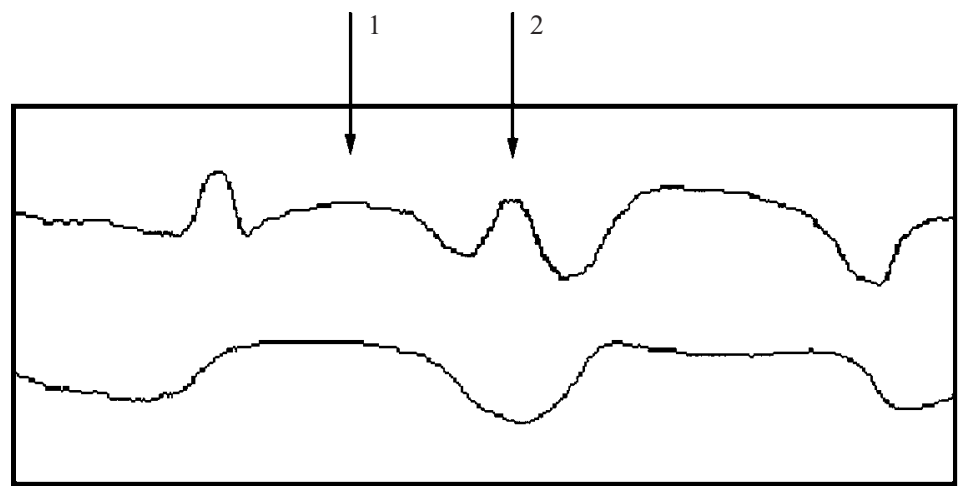

Figure 27. Coexistence of Mode 2 and Mode $1, R e_{1}=220, S t=0.07, x=18 \mathrm{~cm}$.

(a)

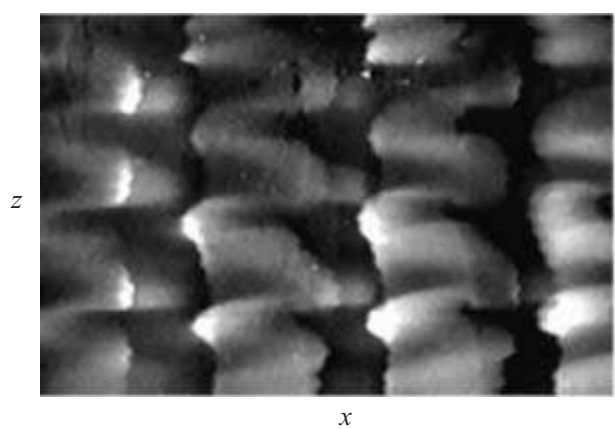

(b)

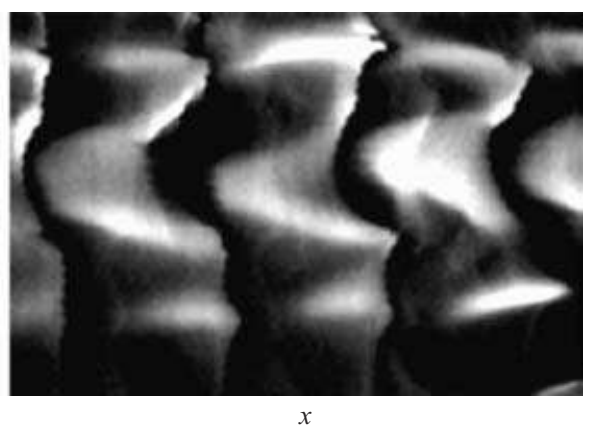

FIGURE 28. Spotlight visualization of two runs at the same experimental conditions, $R e_{2}=200$, $S t=0.07$, taken at the same downstream location $x \approx 10 \mathrm{~cm}$, the flow is from left to right: (a) Mode 2, $(b)$ Mode 1. Both pictures are about the same size in $x\left(\approx 3.5 \times \lambda_{2 D}\right)$, but they have been taken with a different focal and parallax effects. Parallax effects are negligible in (b) and the same scale applies in the $x$-and $z$-directions whereas the scale in the $z$-direction should be multiplied by an approximated factor 1.7 in $(a)$ to be compared with the $x$-direction (and with $b$ ).

Mode 2 results in a complexity of the interface deformations at these downstream locations which do not allow us to study this wake region further.

The coexistence of both modes in the far wake was also confirmed by two experiments performed under exactly the same experimental conditions $\left(R e_{2}=200\right.$ and $S t=0.07$ with the secondary three-dimensional instability unforced) where we observed by chance a strong amplification of Mode 2 in the first run (figure 28a), while in the second run, a strong amplification of Mode 1 was detected (figure 28b).

Figure $28(a, b)$ shows two top-view spotlight visualizations of these two wakes developing under the same conditions. The upper part of the interface alone is shown in these visualizations, the lower part being in the dark zone. In the first run (figure 28a), the streamwise vortices, visualized by filaments of dye connecting the primary rolls, are aligned in the flow direction, a characteristic feature of Mode 2 (compare this figure to figure $3 b$ ), whereas in the second run (figure 28b) the streamwise vortices exhibit a lambda-shape characteristic of Mode 1 (see figure $3 a$ ). The appearance of a pure Mode 1 was an exceptional event but, when it occurred, the order of magnitude for $\lambda_{3 D} / \lambda_{2 D}$ was about 1 . Figure 28 provides evidence that the two 

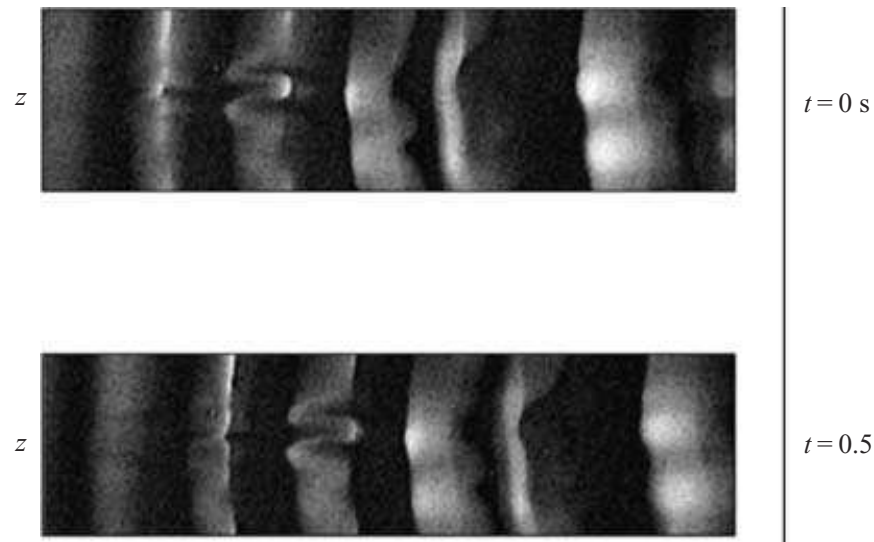

$t=0.5 \mathrm{~s}$

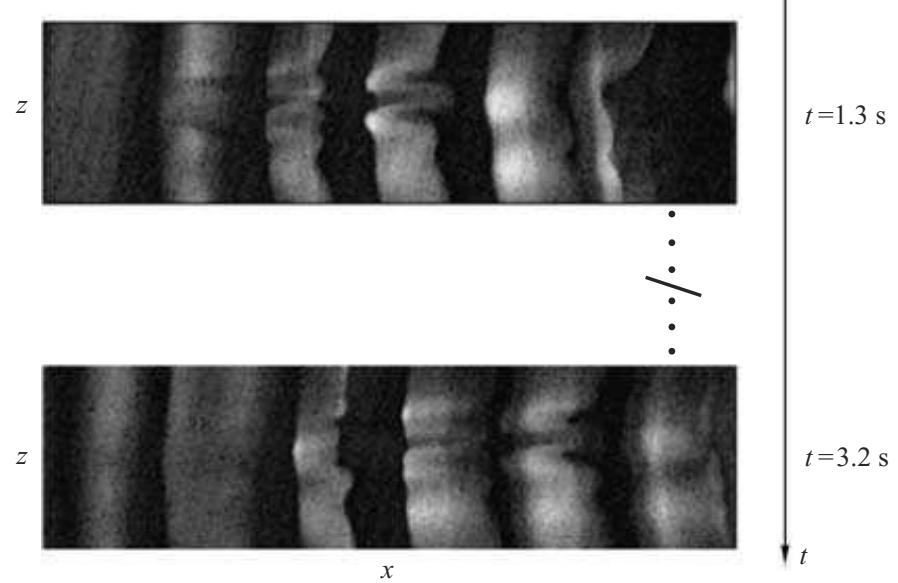

FIGURE 29. Spotlight visualization of the primary vortices: temporal sequence following a localized perturbation, $R e_{2}=200, S t=0.07$. The streamwise length $(x$-axis $)$ is about $29 \mathrm{~cm}$.

modes compete (i.e. have comparable growth rate, Mode 1 being significantly smaller since its observation is rare) and have comparable wavelengths within a $20 \%$ tolerance.

\subsection{Nature of the secondary instability}

Two facts suggest that the secondary instability is convective. First, without any controlled three-dimensional forcing the secondary instability is irregular and depends upon the noise present in the flows. Secondly the flow is strongly receptive to the low-amplitude three-dimensional forcing. This assumption seems to be confirmed by a particular state of the flow that was observed in several independent runs in which the background noise was, by chance, particularly low.

Figure 29 presents a temporal sequence of spotlight visualizations showing the evolution of a localized perturbation that in this case appeared impulsively and randomly. In these pictures, the mean flow is from left to right. The perturbation appears close to the splitter plate and grows while being advected by the flow. Note 
that in the last frame, the flow has returned to the base state at the location where the perturbation was initially detected. Note also that the perturbation develops a varicose structure characteristic of Mode 2 (compare this with the spotlight visualization of figure 28a). This observation is consistent with the behaviour of a convectively unstable flow for which a perturbation localized in space and time gives rise to a wave packet which is amplified as it moves downstream. Meanwhile, the flow relaxes back to the basic state at the location where the perturbation was originally introduced. It should be noted that in the wake of the flat plate studied here, we have observed consistently the above described response to impulsive perturbations, indicating the convective nature of this instability. It should be emphasized that this result may hold true only for wakes forming behind a flat plate and not for separated bluff body wakes. In bluff body wakes, where there is a separated flow region behind the body, Barkley \& Henderson (1996) concluded that the development of the threedimensional instability in the wake is driven by a 'near-wake' instability. This might be the sign of an absolute secondary instability (Huerre \& Monkewitz 1990) although such a conjecture requires further exploration and is outside the scope of the present paper.

\section{Discussion and speculation on the wavelength and mode selection in plane wakes}

All the experiments reported here were limited to planar wakes forming behind a thin flat plate at low Reynolds numbers of 200 and 220. These wakes, which are formed as two parallel laminar streams merge at the trailing edge of a very thin flat plate, possess a single characteristic length, i.e. the boundary layer thickness (or the momentum thickness, or any other dimension which characterizes the state of the laminar boundary layer on each side of the plate at the time they merge). Under these conditions, we have demonstrated that the dominant three-dimensional mode in the near wake is Mode 2, and that its preferred wavelength is always comparable to that of the two-dimensional mode $\left(\lambda_{3 D} / \lambda_{2 D} \sim 1\right)$. When Mode 2 is forced, the most amplified mode wavelength is $\lambda_{3 D} / \lambda_{2 D}=0.8$ and its spatial growth rate is close to $0.35 \mathrm{~cm}^{-1}$. We also found that Mode 1 appears to be slightly less unstable, with a preferred wavelength $\left(\lambda_{3 D} / \lambda_{2 D} \sim 1.1\right)$ approximately the same as Mode 2 . Furthermore, we confirmed the co-existence of both modes under the same Reynolds number regime. This evidence favours the hypothesis that both observed modes are a result of the same type of instability.

Robinson \& Saffman (1982), studying the three-dimensional stability of vortex arrays, show that, when the core diameter of the vortices is small compared to $\lambda_{2 D}$, the Kármán vortex street is unstable to long-wavelength spanwise disturbances for small values of the ratio $b / \lambda_{2 D}$ where $b$ is the distance between the two rows of opposite sign vortices. They found that the dominant mode is anti-symmetric with a most unstable wavelength which depends on the ratio $b / \lambda_{2 D}$ and is such that $\lambda_{3 D} / \lambda_{2 D}$ varies between 3 and 6.5 . Since this mode possesses the symmetry of Mode 1, this analysis does not agree with the present flat-plate wake experiment where we observed that although in some cases the anti-symmetric mode (Mode 1) finally appears, the symmetric mode (Mode 2) appears to dominate. Neither does the theoretical wavelength selection match with the present experimental results. In the Robinson \& Saffman (1982) study, the core of the vortices was not allowed to deform, and only long wavelengths compared to the core size were studied. In all real wakes, the core is not small compared to $\lambda_{2 D}$ and therefore the Robinson \& Saffman 
analysis applies only to long wavelengths compared to $\lambda_{2 D}$. At shorter wavelengths, deformations of the core must be considered and an elliptic instability takes place since each vortex is subjected to the strain fields of the others. This mechanism has been studied for single finite size vortices with uniform vorticity by Tsai \& Widnall (1976), Robinson \& Saffman (1984) and Waleffe (1990). Klassen \& Peltier (1991) and Potylitsin \& Peltier (1999) have shown that this instability is also present in mixing layers. Julien, Chomaz \& Lasheras (2002) studying the Mallier \& Maslowe (1993) wake model for which $b=0$ have shown that elliptic instability leads to both Mode 1 and Mode 2 with similar growth rates.

Following Klaassen \& Peltier's (1991) stability analysis, the braid region in the early stage of the vortex roll-up (i.e. before the braid is depleted by the strain field) exhibits an instability with a length scale of the braid thickness that initially equals the boundary layer thickness (see also Leblanc \& Cambon 1998; Caulfield \& Peltier 2000; Caulfield \& Kerswell 2000): the hyperbolic instability. This hyperbolic instability may also account for the wavelength selection of Mode 1 and Mode 2 but the fact that the wavelength selection observed does not scale on $\bar{\delta}$ but on $\lambda_{2 D}$ does not favour this hypothesis.

The study of the stability of a computed wake model generated by the saturation of a Kármán street formed by the destabilization of the Bickley jet (Sutherland \& Peltier 1994) predicts that both Mode 1 and Mode 2 have similar growth rates with a maximum for a wavelength close to 0.8 , a result in remarkable agreement with experimental observations reported here. In their study, they have shown that the perturbation extracts energy concomitantly from the braid and from the core regions of the flow. Although their study, focused on the stratification effect on the transition to turbulence in a jet, has only reported results at time $t=50$ for $\lambda_{2 D}=\pi \bar{\delta}$, it clearly indicates that both the braid and the core are responsible for the secondary instability of a Bickley wake no matter what its symmetry is. In other words, the mechanism common to both modes is a combination of the hyperbolic instability due to the stretching at the stagnation point and the elliptic instability of the primary vortex cores.

In the flat-plate wake where both instabilities are associated with similar scale selection, the elliptic instability of the two-dimensional Kármán vortices or the hyperbolic instability of the braid regions may explain the present results. However, the question which remains to be investigated is whether these results are also applicable to wakes behind bluff bodies where the shear layers separate at a certain location on the surface of the body, thus introducing a second characteristic length in the problem. Comparison of our results to recent cylinder wake experiments is enlightening (see Williamson $1996 b$ for a review). In the case of the wake behind a cylinder Mode A, which develops when the Reynolds number is above a well-defined threshold $\left(R e_{c} \approx 190\right)$, possesses the symmetry of our Mode 2 and its wavelength $\left(\lambda_{3 D} / \lambda_{2 D}\right)$ is in the range $[3 / 5,4 / 5]$, thus close to our values of around 0.8 , despite the strong difference between the two types of wakes. On the other hand, Mode B which possesses the symmetry of Mode 1 is observed in the cylinder case at higher Reynolds number $\left(R e_{c} \approx 260\right)$, and is found to have a wavelength four times smaller, $\lambda_{3 D} / \lambda_{2 D} \approx 1 / 4$. It is important to mention that such a small wavelength was never observed in our flat-plate experiments for the Reynolds numbers cases studied here. However, note that for the flat plate, the Reynolds number was based on the Bickley wake profile, whereas for the cylinder wake it is based on the diameter. Leweke \& Williamson (1998) argued that in the cylinder configuration, Mode A which has a wavelength comparable to $\lambda_{2 D}$ is a result of an elliptic instability of the two-dimensional Kármán vortices and Mode $\mathrm{B}$ is generated by 
the hyperbolic instability of the braid regions. The core and the braid instabilities might account for the wavelength selection in the cylinder and plate wakes but not for the eventual symmetry selection as proposed by Leweke \& Williamson (1998) since in the flat-plate wake Mode 1 and Mode 2, which possess the same symmetry characteristics as Mode B and Mode A, present a wavelength scaling on the twodimensional wavelength, indicating that both modes result from the same instability mechanism.

The search for an interpretation of the differences between bluff bodies and plate wakes from local stability theory continues. Success in this regard would ultimately allow a precise identification of the different instability mechanisms and the associated wavelength selection with respect to particular features of the vorticity distribution such as vortex concentration and spacing between eddies as in Robinson \& Saffman (1982). Such studies have been successfully performed for stratified mixing layers by Klaassen \& Peltier (1991) and Potylitsin \& Peltier (1999). For the flat-plate wake, the local stability analysis is pertinent since the primary and the secondary instabilities develop slowly and a numerical estimate of the leading local eigenmodes explains both the wavelength selection and the growth rate observed (Sutherland \& Peltier 1994; Julien 2000; Julien et al. 2002). The cylinder wake is far more complex since the base flow varies rapidly behind the cylinder. Barkley \& Henderson (1996) have shown, from a stability analysis of the non-parallel base flow, that the secondary instability observed in the experiments is due to a global secondary bifurcation. Both the primary base flow and the secondary global modes grow very quickly in space. This invalidates a weakly non-parallel approximation and the connection of the global secondary instability with the local property of the flow is therefore lost. However, it would still be interesting to address the link between the global instability of the fully non-parallel base flow computed by Barkley \& Henderson (1996) and a possible absolute secondary instability of the associated parallel wake flow. This would be consistent with the observations from experiments. Indeed, the secondary instability in the wake of a flat plate, which has no back flow and a vorticity distributed in large patches, is found to be convective. Unlike this, the cylinder wake is characterized by a region behind the cylinder where the flow reverses and where the vorticity is highly concentrated. In this region, the primary instability is absolutely unstable and this explains the occurrence and the frequency selection of the primary global instability (Huerre \& Monkewitz 1991; Monkewitz 1988). It would be natural that the same features will favour the absolute instability nature of the secondary instability and may explain the observed and computed thresholds and wavelength selection of Mode B and eventually Mode A.

\section{Conclusion}

We have studied the development of the secondary instability in the wake forming as two parallel laminar streams merge at the trailing edge of a thin flat plate. Analysis of the symmetry properties of the interface deformation revealed that Mode 2 always grows first, dominating the evolution of the near wake. Measurements conducted in the naturally evolving flow as well as that from forcing the primary two-dimensional instability over a wide range of frequencies revealed that the wavelengths of both the two-dimensional and three-dimensional modes are always comparable $\left(\lambda_{3 D} / \lambda_{2 D} \approx 1\right)$. We have also shown that both modes can develop and co-exist in the far wake under the same Reynolds number regime. Furthermore, we found that they have similar wavelengths which scale with the wavelength of the primary instability. This result 
was further confirmed by measurements of the growth rate of the three-dimensional modes developing for a wide range of spanwise forcing wavelengths introduced at the trailing edge of the flat plate. It reaches a maximum for $\lambda_{3 D} / \lambda_{2 D}=0.76$ with a growth rate close to $0.37 \mathrm{~cm}^{-1}$. These results are in remarkable agreements with Sutherland \& Peltier (1994) who predict that, in the Bickley wake, both Mode 1 and Mode 2 have similar growth rates with a maximum for a wavelength close to 0.77. In their study, they have shown for both modes that the perturbation extracts energy from the braid region and from the vortex core. It appears to indicate that the three-dimensional modes are due to the same instability mechanism, a combination of the hyperbolic instability at the stagnation point between consecutive vortices and the elliptic instability of the primary vortices.

Finally, the response of the wake to an impulsive three-dimensional perturbation demonstrates the convective nature of the secondary instability developing in the wake of the thin flat plate.

The authors would like to thank warmly Marcus Lobbia who assisted with performing all the experiments, Ivan Delbende who initiated the numerical work to justify the validity of the growth rate measurements and C. P. Caulfield who provided useful comments on the revised version of this paper.

\section{REFERENCES}

BARKLEy, D. \& Henderson, R. D. 1996 Three-dimensional Floquet stability analysis of the wake of a circular cylinder. J. Fluid Mech. 322, 215.

Brancher, P. 1996 Numerical and theoretical investigation of jet instabilities. PhD thesis, École Polytechnique.

BraZA, M. 1994 Transition features in wake flows by means of numerical analysis. In Current Topics in the Physics of Fluids (ed. J. Mener), p. 391. Council of Scientific Information, Trivandrum, India.

Caulfield, C. P. \& Kerswell, R. R. 2000 The nonlinear development of three-dimensional disturbances at hyperbolic stagnation points: A model of the braid region in mixing layers. Phys. Fluids 12, 1032.

Caulfield, C. P. \& Peltier, W. R. 2000 The anatomy of the mixing transition in homogeneous and stratified free shear layers, J. Fluid Mech. 413, 1.

Cimbala, J. M., Nagib, H. M. \& Roshko, A. 1988 Large structure in the far wakes of twodimensional bluff bodies. J. Fluid Mech. 190, 265.

Delbende, I. \& Chomaz, J.-M. 1998 Nonlinear convective/absolute instabilities of parallel twodimensional wakes. Phys. Fluids 10, 2724.

Drazin, P. G. \& Howard, L. N. 1966 Hydrodynamic stability of parallel flow of inviscid fluid. Adv. Appl. Mech. 9, 1.

Drazin, P. G. \& ReID, W. H. 1981 Hydrodynamic Stability, p. 233. Cambridge University Press.

EISENlohr, H. \& ECKelmann, H. 1989 Vortex splitting and its consequences in the vortex street wake of cylinders at low Reynolds numbers. Phys. Fluids A 1, 189.

Ferre, J. A. \& Girault, F. 1989 Pattern-recognition analysis of the velocity field in plane turbulent wakes. J. Fluid Mech. 198, 27.

GASTER, M. 1964 A note on the relation between temporally-increasing and spatially-increasing disturbances in hydrodynamic stability, J. Fluid Mech. 14, 222.

Grant, M. L. 1958 The large eddies of turbulent motion. J. Fluid Mech. 4, 149.

Hama, R. 1962 Streaklines in a perturbed shear flow. Phys. Fluids 5, 644.

Huerre, P. \& Monkewitz, P. A. 1990 Local and global instabilities in spatially developing flows. Annu. Rev. Fluid Mech. 22, 473.

Julien, S. 2000 Instabilités secondaires dans les sillages plans. PhD thesis, École Polytechnique.

Julien, S., Chomaz, J.-M. \& Lasheras, J. C. 2002 Study of the temporal stability of a row of counter-rotating vortices. Phys. Fluids 14, 732. 
Julien, S., Ortiz, S. \& Chomaz, J.-M. 2002 The three-dimensional instabilities in the wake of a flat plate. Phys. Fluids (submitted).

Klatassen, G. P. \& Peltier, W. R. 1991 The influence of stratification on secondary instability in free shear layers. J. Fluid Mech. 227, 71.

König, M., Eisenlohr, H. \& Eckelman, H. 1990 The fine structures in the $S$-Re relationship of the laminar wake of a circular cylinder. Phys. Fluids A 2, 1607.

KöNig, M., EISEnlohr, H. \& ECKelman, H. 1992 Visualisation of the spanwise cellular structure of the laminar wake of wall-bounded circular cylinders. Phys. Fluids A 4, 869.

Lasheras, J. C., Сho, J. S. \& Maxworthy, T 1986 On the origin and evolution of streamwise vortical structures in a plane, free shear layer. J. Fluid Mech. 172, 231.

Lasheras, J. C. \& ChOI, H. 1988 Three-dimensional instability of a plane free shear layer. An experimental study of the formation and evolution of streamwise vortices. J. Fluid Mech. 189, 53.

Lasheras, J. C. \& Meiburg, E. 1990 Three-dimensional vorticity modes in the wake of a flat plate. Phys. Fluids A 5, 371.

Leblanc, S. \& Cambon, C. 1998 Effects of the Coriolis force on the stability of Stuart's vortices. J. Fluid Mech. 356, 353.

Leweke, T. \& Williamson, C. H. K. 1998 Three-dimensional instabilities in wake transition. Eur. J. Mech. B/Fluids 17, 571.

Mallier, R. \& Maslowe, S. A. 1993 A row of counter-rotating vortices. Phys. Fluids 5, 1074.

Marasli, B., Champagne, F. H. \& Wygnanski, I. J. 1989 Modal decomposition of velocity signals in a plane, turbulent wake. J. Fluid Mech. 198, 255.

Meiburg, E. \& Lasheras, J. C. 1987 Comparison between experiments and numerical simulations of three-dimensional plane wakes. Phys. Fluids 30, 623.

Meiburg, E. \& Lasheras, J. C. 1988 Experimental and numerical investigation of the threedimensional transition in plane wakes. J. Fluid Mech. 190, 1.

Miller, G. D. \& Williamson, C. H. K. 1994 Control of three-dimensional phase dynamics in a cylinder wake. Exps. Fluids 18, 26.

Monkewitz, P. A. 1988 A note on vortex shedding from axisymmetric bluff bodies. J. Fluid Mech. 192, 561.

Monkewitz, P. A., Williamson, C. H. K. \& Miller, G. D. 1996 Phase dynamics of Kármán vortices in cylinder wakes. Phys. Fluids $\mathbf{8}, 91$.

Mumford, J. C. 1983 The structure of the large eddies in fully turbulent shear flows. Part 2. The plane wake. J. Fluid Mech. 137, 447.

Noack, B. N. \& ECKelmann, H. 1994 A global stability analysis of the steady and periodic cylinder wake. J. Fluid Mech. 270, 297.

Payne, F. \& Lumley, J. L. 1967 Large eddy structure of the turbulent wake behind a circular cylinder. Phys. Fluids Suppl. S194.

Persillon, H. \& BrazA, M. 1998 Physical analysis of the transition to turbulence in the wake of a circular cylinder by three-dimensional Navier-Stokes simulation. J. Fluid Mech. 365, 23.

Pierrehumbert, R. T. \& Widnall, S. E. 1982 The two- and three-dimensional instabilities of a spatially periodic shear layer. J. Fluid Mech. 114, 59.

Potylitsin, P. G. \& Peltier, W. R. 1999 Three-dimensional destabilization of Stuart vortices: the influence of rotation and ellipticity. J. Fluid Mech. 387, 205.

Robinson, A. C. \& Saffman, P. G 1982 Three-dimensional stability of vortex arrays. J. Fluid Mech. 125, 411.

Robinson, A. C. \& Saffman, P. G. 1984 Three-dimensional stability of an elliptical vortex in a straining field. J. Fluid Mech. 142, 451.

Roshko, A. 1954 On the development of turbulence wakes from vortex streets. NACA Rep. 1191.

Schlichting, H. 1930 Über das ebene Windschattenproblem. Diss. Göttingen 1930. Ing.-Arch. 1, 533.

Schowalter, D. G., Van Atta, C. W. \& Lasheras, J. C. 1994 A study of streamwise vortex structure in a stratified shear layer. J. Fluid Mech. 281, 247.

Sutherland, B. R. \& Peltier, W. R. 1994 Turbulence transition and internal wave generation in density stratified jets. Phys. Fluids 6, 1267.

TANEDA, S. 1977 Visual study of unsteady separated flows around bodies. Prog. Aerospace Sci. 17, 287. 
Townsend, A. A. 1979 Flow patterns of large eddies in a wake and in a boundary layer. J. Fluid Mech. 95, 515.

TsaI, C.-Y. \& Widnall, S. E. 1976 The stability of short waves on a straight vortex filament in a weak externally imposed strain field. J. Fluid Mech. 73, 721.

WALEFFe, F. 1990 On the three-dimensional instability of strained vortices. Phys. Fluids A 2, 76.

Williamson, C. H. K. 1988 The existence of two stages in the transition to three-dimensionality of a cylinder wake. Phys. Fluids 31, 3165.

Williamson, C. H. K. 1989 Oblique and parallel modes of vortex shedding in the wake of a circular cylinder at low Reynolds numbers. J. Fluid Mech. 206, 579-627.

Williamson, C. H. K. $1996 a$ Three-dimensional wake transition. J. Fluid Mech. 328, 345.

Williamson, C. H. K. $1996 b$ Vortex dynamics in the cylinder wake. Annu. Rev. Fluid Mech. 28, 477.

Winant, C. D. \& Browand, F. K. 1974 Vortex pairing: the dynamics of turbulent mixing layer growth at moderate Reynolds number. J. Fluid Mech. 63, 237. 